\title{
A novel approach of linguistic intuitionistic cubic hesitant variables and their application in decision making
}

\author{
Muhammad Qiyas $^{1} \cdot$ Saleem Abdullah $^{1} \cdot$ Muneeza $^{1}$
}

Received: 14 October 2019/ Accepted: 27 March 2020/Published online: 25 April 2020

(C) The Author(s) 2020

\begin{abstract}
In this paper, we proposed the notion of linguistic intuitionistic cubic hesitant variables and defined some aggregation operators to deal with uncertainties in the form of linguistic intuitionistic cubic hesitant variables (LICHVs). LICHVs operators have more flexibility due to the general fuzzy set. We developed a series of aggregation operators, namely linguistic intuitionistic cubic hesitant variable averaging and linguistic intuitionistic cubic hesitant variable geometric aggregation operators. The distinguished feature of the developed operators is discussed. At that point, we used the developed operators to design a model to solve multi-criteria decision making issues with linguistic intuitionistic cubic hesitant variables. Further, the proposed method applied to explosion incident occurred in a chemical factory. We also proved that our developed model is practical and gives the decision makers more mathematical insight during the decision making on their options. Finally, a systematic comparison is conducted with other existent methods to show the advantage of our developed method.
\end{abstract}

Keywords Linguistic intuitionistic cubic hesitant variable $\cdot$ Least common multiple number $\cdot$ Weighted average aggregation operator $\cdot$ Weighted geometric aggregation operator

\section{Introduction}

Decision making (DM) is an important research topic in daily activities, such as economic, engineering, education, and medical. In the DM method, a problem involves many sources of information, giving the final result via aggregating process. Due to the complexity of management information and decision problems themselves, decision makers may provide their ratings or judgments to some certain grade, but it is possible that they are not so sure about their judgments. Namely, there may exist some hesitancy grade, which is a very important factor to be taken into account when trying to construct really adequate

Muhammad Qiyas

muhammadqiyas@awkum.edu.pk

Saleem Abdullah

saleemabdullah@awkum.edu.pk

Muneeza

muneeza0315@gmail.com

1 Department of Mathematics, Abdul Wali Khan University Mardan, Mardan, KP, Pakistan models and solutions of decision problems. Such a kind of hesitancy grade is suitably expressed with Zadeh fuzzy sets (1965), rather than exact numerical values, and applied in many fields (Chen et al. 1992, 2012, 2013; Chen and Tsai 2008; Chen and Huang 2014; Chen and Chen 2014). After that, the notion of intuitionistic fuzzy sets (IFSs) introduced by Atanssove (1986) is the generalized form of Zadeh fuzzy sets (FSs). Each number of an IFS is represented by an ordered pair consisting of positive and negative membership grades, where the sum of both positive membership and negative membership grade is less than or equal to one, and thus can depict the fuzzy character of data more detailed and comprehensively than fuzzy set which is characterized by a positive membership grade only. Several researchers (Chen et al. 2016; Liu et al. 2017, 2018; Liu and Chen 2018) have done quite valuable contribution in the development of IFS and its applications, the result of which is in the form of great success of IFSs in theoretical and technical aspects. Bai and Chen (2008) developed automatically constructing grade membership functions of fuzzy rules for students' evaluation. Bai and Chen (2008) defined an automatically constructing concept maps based 
on fuzzy rules for adapting learning systems. Chen (1996) defined a fuzzy reasoning approach for rule-based systems based on fuzzy logics.

A major part of MCGDM with IFSs is the aggregation of intuitionistic fuzzy information (Shuqi et al. 2009; Zhao et al. 2010; Li 2010; Li and Wu 2010; Li 2011; Nayagam et al. 2011; Zhou et al. 2013; Zhou and Chen 2014; Kou et al. 2016; Liang et al. 2017; Ye 2018; Garg and Kumar 2019). The decision making process under undetermined or firm circumstances and intuitionistic fuzzy numbers (IFNs) are too much convenient to disclose sensitive information of a decision maker over objects. The aggregation of IFNs is an essential step to get a decision problem's outcome. For this purpose, a number of operators have been introduced recently to aggregate IFNs which are known as intuitionistic fuzzy hybrid aggregation (IFHA) operator, intuitionistic fuzzy hybrid geometric (IFHG) operator, intuitionistic fuzzy ordered weighted averaging (IFOWA) operator, intuitionistic fuzzy ordered weighted geometric (IFOWG) operator, intuitionistic fuzzy weighted averaging (IFWA) operator, and intuitionistic fuzzy weighted geometric (IFWG) operator (Kim and Ahn 1999; Beliakov et al. 2011; Yager et al. 2011; Yang and Yuan 2014; Garg 2017; Gitinavard et al. 2017; Liu and Liu 2017; Rani and Garg 2018; Shakeel 2018).

However, IFS does not explain the uncertainty problems. To overcome this difficulty, Jun et al. (2011) introduced cubic fuzzy set (CFS). This theory made it possible to deal with uncertainty problems. Cubic set theory also explains the satisfied, unsatisfied, and unpredictable information, which were not explained by FS theory and IFS theory (Mahmood et al. 2016; Fahmi et al. 2017, 2018a, b, c, d, e, f; Kaur and Garg 2019; Riaz and Tehrim 2019; Fahmi et al. 2019). Cubic set has more desirable information than FS and IFS (Kaur and Garg 2018a; Kaur and Garg 2018b). It is one of the generalized forms of FS and IFS; just like IFS, every element of a cubic fuzzy set is represented as a structure of an ordered pair, which is characterized by function of membership and function of non-membership. The non-membership is just like the normal fuzzy set, whereas the function of membership is grip in the form of an interval.

Phong and Cuong (2015) and Herrera and HerreraViedma (2000) proposed an algorithm for solving the linguistic decision making problems. Next, Xu (2004a) put forward linguistic aggregation operators, like as linguistic geometric average, linguistic weighted geometric average, linguistic ordered weighted geometric average, and linguistic hybrid geometric average operators, for group decision making with linguistic preference relations, and then Xu (2006a) developed a linguistic hybrid average operator for linguistic multi-attribute group decision making. However, linguistic information provided by decision makers may be uncertain due to the uncertainty of decision environment and lack of decision makers' knowledge. Hence, Xu (2004b) proposed an uncertain linguistic ordered weighted averaging and uncertain linguistic hybrid aggregation operators and applied them to uncertain linguistic group decision making. Further, Xu (2006b) introduced induced uncertain linguistic ordered weighted average operators for uncertain linguistic group decision making problems. Wei (2009) presented an uncertain linguistic hybrid geometric mean operator and applied it to multi-attributes group decision making with uncertain linguistic preference relations. Moreover, some researchers (Park et al. 2011; Wei et al. 2013; Zhang 2015) also proposed an uncertain linguistic Bonferroni mean operator, uncertain linguistic power geometric operators, and uncertain linguistic harmonic mean operators for decision making under uncertain linguistic environments.

However, linguistic hesitant fuzzy set does not explain the uncertainty problems. To overcome this difficulty, Jun and Cui (2018) introduced linguistic cubic hesitant variables. This theory made it possible to deal with uncertainty problems. Linguistic cubic hesitant variable theory also explains the satisfied, unsatisfied, and uncertain information, which were not explained by linguistic intuitionistic fuzzy set theory. Linguistic cubic hesitant variable (LCHV) has more desirable information than linguistic fuzzy set and linguistic intuitionistic fuzzy set (LIFS). Linguistic cubic hesitant variables are the generalizations of linguistic fuzzy sets and linguistic cubic variables; just like LIFS, each element of linguistic cubic variable is represented by an ordered pair, which is characterized by linguistic membership grade and linguistic non-membership grade. The positive membership grade is grip in the form of interval, whereas the negative membership is just like the normal fuzzy set.

Due to the motivation and inspiration of the above discussion in this paper, we have given a new approach of LICHV through application of cubic set theory. For instance, introduce the concept of linguistic intuitionistic cubic hesitant variable (LICHV). Each element of which consists of a function of linguistic membership and linguistic non-membership. Linguistic membership function is a cubic fuzzy set, and linguistic non-membership function is also a cubic fuzzy set. LICHV is the hybrid set which can contain much more information to express a LCFS and an LIFS simultaneously, for handling the uncertainties in the data.

The highlights of the developed approach are the following:

(1) We first time extends the existing concept of LCHVs to the LICHVs, to express interval/uncertain linguistic and hesitant linguistic arguments, respectively. 
(2) We proposed the weighted average and geometric aggregation operators for the LICHVs, using the least common multiple number (LCMN) extension method, and defined the linguistic score function for the LICHVs.

(3) We proposed a MCDM algorithm with LICHVs, which is the extension of the existing MCDM algorithm with LCHVs, so as to carry out the MCDM problems with the hybrid information of both interval linguistic arguments and hesitant linguistic arguments, which cannot be handled by the existing ones .

The remainder of the article is as follows: In Sect. 2, we briefly discussed the basic knowledge about the fuzzy set, intuitionistic fuzzy set and linguistic cubic variables. In Sect. 3, we present some operational laws for linguistic intuitionistic cubic hesitant variables and their score function. In Sect. 4, we present average and geometric aggregation operators of linguistic intuitionistic cubic hesitant variables. Utilizing the weighted averaging (WA) and weighted geometric (WG) operators, we proposed a model for multi-criteria decision making problem in Sect. 5. In Sect. 6, some discussions are made on the application of the developed method and its comparison with existing approaches and finally presented the conclusion in Sect. 7.

\section{Preliminaries}

In this section, some basic knowledge about the fuzzy set, intuitionistic fuzzy set, linguistic intuitionistic fuzzy variables and their precious properties is discussed.

Definition 1 Zadeh (1965) Suppose that $\mathbb{R} \neq \phi$ be a set. A fuzzy set $\mathfrak{R}$ in $\mathbb{R}$ is defined as:

$\mathfrak{R}=\left\{\left(\dot{r}^{\prime}, \mu_{\mathfrak{R}}\left(\dot{r}^{\prime}\right)\right) \mid \dot{r}^{\prime} \in \mathbb{R}\right\}$,

where $\mu_{\mathfrak{R}}: \mathbb{R} \rightarrow[0,1]$ is the membership grade of a fuzzy set $\mathfrak{R}$.

Definition 2 Atanasav (1986) Suppose that $\mathbb{R} \neq \phi$ be a set. An intuitionistic fuzzy set $\mathfrak{R}$ in $\mathbb{R}$ is defined as:

$\mathfrak{R}=\left\{\left(\dot{r}^{\prime}, \mu_{\mathfrak{R}}(\dot{r}), v_{\mathfrak{R}}(\dot{r}) \mid \dot{r}^{\prime} \in \mathbb{R}\right\}\right.$,

where the functions $\mu_{\mathfrak{R}}\left(\hat{r}^{\prime}\right): \mathbb{R} \rightarrow[0,1]$ and $v_{\mathfrak{R}}(\hat{r}): \mathbb{R} \rightarrow$ $[0,1]$ denote the positive and negative membership grades of each number $r \in \mathbb{R}$, respectively, with $0 \leq \mu_{\mathfrak{R}}\left(r^{\prime}\right)+$ $v_{\mathfrak{R}}(\hat{r}) \leq 1$ for all $\dot{r} \in \mathbb{R}$.

Furthermore, we have $\pi_{\mathfrak{R}}\left(\dot{r}^{\prime}\right)=1-\mu_{\mathfrak{R}}\left(\dot{r}^{\prime}\right)-v_{\mathfrak{R}}\left(r^{\prime}\right)$, called intuitionistic fuzzy index or hesitation margin of $r$ to $\mathfrak{R S z m i d t}$ and Kacprzyk (2000). $\pi_{\mathfrak{R}}\left(r^{\prime}\right)$ is also called the grade of indeterminacy of $r^{\prime} \in \mathbb{R}$ to the IFS $\mathfrak{R}$.
Definition 3 Jun et al. (2011) A linguistic cubic variable (LCVs) $\mathfrak{R}$ in $\mathbb{R} \neq \phi$ is given as the following:

$\mathfrak{R}=\left\{s_{\mu}, s_{v}\right\}$

where the first element is an LCV denotes the grade of linguistic membership and the second is a simple linguistic fuzzy number.

\section{Linguistic intuitionistic cubic hesitant variables and their operational laws}

Definition 4 Herrera and Herrera-Viedma (2000) Let $S=$ $\left(s_{0}, s_{1}^{\prime}, \ldots, s_{\ell-1}^{\prime}\right)$ be the finite and absolutely order distinct term set. Then, $S$ is the linguistic term set, where $\ell$ is the even value, e.g., $3,5, \ldots$, when $\ell=5$, then $S$ can be written as $S^{\prime}=\left(s_{0}^{\prime}, s_{1}, s_{2}, s_{3}, s_{4}^{\prime}\right)=$

(poor, slightly poor, fair, slightly good, good).

The following characteristics of the linguistic set $S$ must be satisfied:

(1) Ordered : $s_{l}^{\prime} \prec s_{l}^{\prime}, \Leftrightarrow l \prec l$;

(2) Negation : neg $\left(s_{l}^{\prime}\right)=s_{\ell-1-l}$;

(3) Maximum: $\left(s_{l}^{\prime}, s_{l}^{\prime}\right)=s_{l}^{\prime}$, iff $\imath \geq l$;

(4) Minimum: $\left(s_{l}^{\prime}, s_{l}^{\prime}\right)=s_{l}^{\prime}$, iff $l \leq l$.

The extended form of the discrete term set $S$ is called a continuous linguistic term set and defined as $S^{*}=$ $\left\{s_{\psi} \mid s_{0} \leq s_{\psi} \leq s_{g}^{\prime}, \psi \in[0, \ell]\right.$, and if $s_{\psi}^{\prime} \in S^{*}$, then $s_{\psi}^{\prime}$ is said to be original term, otherwise virtual term.

Definition 5 A linguistic cubic variable $\mathfrak{R}$ in $\mathbb{R} \neq \phi$ is given as the following:

$\mathfrak{R}=\left\{s_{\mu}^{\prime}, s_{h}\right\}$,

where $s_{\mu}^{\prime}=\left[s_{\mu^{-}}, s_{\mu^{+}}\right]$for $\mu^{-} \geq \mu^{+}$and $\dot{s}_{\mu^{-}}, \dot{s}_{\mu^{+}} \in S^{*}$ is an internal linguistic variable and $s_{h}=$ $\left\{s_{\lambda_{\kappa}} \mid \dot{s}_{\lambda_{\kappa}} \in S^{\prime}, \kappa=1, \ldots, p\right\}$ is a set of $p$ possible linguistic variables (i.e., hesitant linguistic variables).

Definition 6 Let $S^{*}=\left\{s_{\psi} \mid s_{0} \leq s_{\psi} \leq s_{g}, \psi \in[0, \ell]\right.$, be a continuous linguistic term set (CLTS). Then, a linguistic intuitionistic cubic hesitant variable (LICHV) is defined as follows:

$\mathfrak{R}=\left\{\left\langle s_{\mu}^{\prime}, s_{\phi}^{\prime}\right\rangle,\left\langle s_{v}^{\prime}, s_{\chi}^{\prime}\right\rangle\right\}$,

where $s_{\mu}^{\prime}=\left[s_{\mu^{-}}, s_{\mu^{+}}\right] \quad$ and $\quad s_{v}^{\prime}=\left[s_{v^{-}}, s_{v^{+}}\right]$for $\mu^{+}$ $\geq \mu^{-}, v^{+} \geq v^{-}$and $\left[\dot{s}_{\mu^{-}}, \dot{s}_{\mu^{+}}^{\prime}, \dot{s}_{v^{-}}, \dot{s}_{v^{+}}\right] \in S^{*}$ are the uncertain linguistic numbers and $\left(\dot{s}_{\phi}^{\prime}, s_{\chi}^{\prime}\right)=\left\{\dot{s}_{\lambda_{\kappa}} \mid s_{\lambda_{\kappa}} \in S^{*}, \quad \kappa=\right.$ $1, \ldots, p\}$ are the set of LVs (i.e., hesitant linguistic variables). If $\lambda_{\kappa} \in\left[\mu^{+}, v^{+}\right](\kappa=1, \ldots, p)$, then $\mathfrak{R}=$ $\left\{\left\langle s_{\mu}, s_{\phi}\right\rangle,\left\langle s_{v}^{\prime}, s_{\chi}\right\rangle\right\}$ is an internal LICHVs, and if $\lambda_{\kappa} \notin$ 
$\left[\mu^{+}, v^{+}\right](\kappa=1, \ldots, p)$, then $\mathfrak{R}=\left\{\left\langle\dot{s}_{\mu}, s_{\phi}^{\prime}\right\rangle,\left\langle s_{v}^{\prime}, s_{\chi}^{\prime}\right\rangle\right\}$ is an external LICVs.

Let we have two LICHVs, $\mathfrak{R}_{1}=\left\{\left\langle\dot{s}_{\mu_{1}}, \dot{s}_{\phi_{1}}\right\rangle,\left\langle s_{v_{1}}, s_{\chi_{1}}\right\rangle\right\}$ and $\mathfrak{R}_{2}=\left\{\left\langle\dot{s}_{\mu_{2}}, \dot{s}_{\phi_{2}}\right\rangle,\left\langle\dot{s}_{v_{2}}, \dot{s}_{\chi_{2}}\right\rangle\right\}$; the number of LVs in hesitant term may be different. Then, to realize the suitable operations of different LICHVs, we used the least common multiple number (LCMN) extension method, to extend the HLV terms until they both reach the same number of LVs. For this:

Assume that $\mathfrak{R}_{\jmath}=\left\{\left\langle\left[\dot{s}_{\mu_{\jmath}^{-}}, s_{\mu_{\jmath}^{+}}\right],\left(\dot{s}_{\lambda_{11}}, s_{\lambda_{\jmath 2}}, \ldots, s_{\lambda_{j p}}\right)\right\rangle\right.$, $\left.\left\langle\left[s_{v_{j}^{-}}, s_{v_{j}^{+}}\right],\left(s_{\lambda_{11}}, s_{\lambda_{\jmath 2}}, \ldots, s_{\lambda_{j p}}\right)\right\rangle\right\}(\jmath=1, \ldots, n)$ are the set of LICHVs and LCMN of $\left(\dot{r}_{1}, \ldots, \dot{r}_{n}\right)$ for $\left(\dot{s}_{\phi_{j}}, s_{x_{\jmath}}\right)(\jmath=$ $1, \ldots, n)$ is $c$. Then, the following extension forms are used to extend them to the same number of linguistic variables:

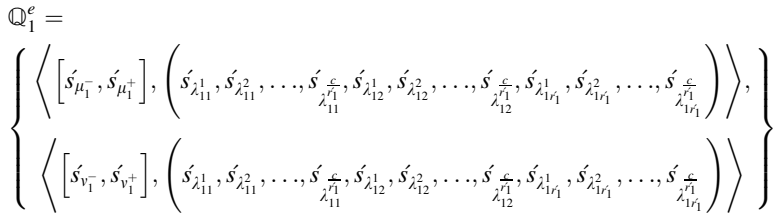

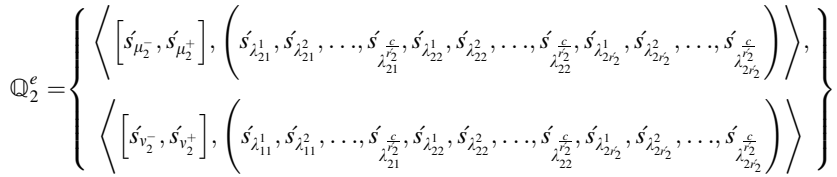

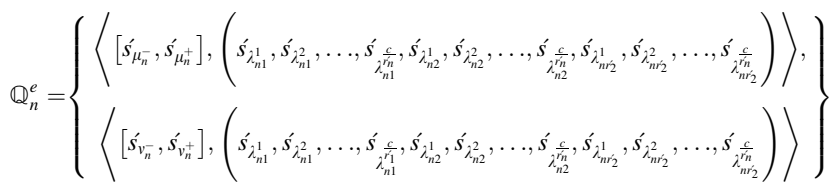

$\left.\left\langle\left[\dot{s}_{v^{-}}, \dot{s}_{v^{+}}\right],\left(\dot{s}_{\lambda_{1}}, \dot{s}_{\lambda_{2}}, \ldots, s_{\lambda_{2}}\right)\right\rangle\right\}$ be a LICHV in the CLTS $S^{*}=\left\{s_{\psi} \mid s_{0} \leq \dot{s}_{\psi} \leq \dot{s}_{g}, \psi \in[0, \ell]\right.$. Then, the linguistic score function is defined as:

$S c(\mathfrak{R})=s_{\frac{1}{4}\left(\frac{1}{4}(\mu+v)+\frac{1}{p} \sum_{k=1}^{p} \lambda_{\kappa}\right.}$ for $S c(\mathfrak{R}) \in[0, \ell]$.

\section{Definition 8 Let}

$$
\begin{gathered}
\mathfrak{R}_{1}=\left\{\left\langle\left[s_{\mu_{1}^{-}}, s_{\mu_{1}^{+}}\right],\left(s_{\lambda_{11}}, s_{\lambda_{12}}, \ldots, s_{\lambda_{1 p}}\right)\right\rangle,\right. \\
\left.\left\langle\left[s_{v_{1}^{-}}^{\prime}, s_{v_{1}^{+}}\right],\left(s_{\lambda_{11}}, s_{\lambda_{12}}, \ldots, s_{\lambda_{1 p}}\right)\right\rangle\right\}
\end{gathered}
$$

and $\quad \mathfrak{R}_{2}=\left\{\left\langle\left[\dot{s}_{\mu_{2}^{-}}^{\prime}, s_{\mu_{2}^{+}}\right],\left(s_{\lambda_{21}}^{\prime}, \dot{s}_{\lambda_{22}}, \ldots, s_{\lambda_{2 p}}\right)\right\rangle, \quad\left\langle\left[s_{v_{2}^{-}}^{\prime}, \dot{s}_{v_{2}^{+}}\right]\right.\right.$, $\left.\left.\left(s_{\lambda_{21}}, s_{\lambda_{22}}, \ldots, s_{\lambda_{2 p}}\right)\right\rangle\right\}$ be the two linguistic intuitionistic cubic hesitant variables in the CLTS $s^{*}=\left\{s_{\psi} \mid s_{0} \leq \dot{s}_{\psi} \leq \dot{s}_{g}, \psi \in[0, \ell]\right.$. Then, their comparison rules on the score values are defined as:
. If $\operatorname{Sc}\left(\mathfrak{R}_{1}\right)>S c\left(\mathfrak{R}_{2}\right)$, then $\mathfrak{R}_{1}>\mathfrak{R}_{2}$;

. If $S c\left(\mathfrak{R}_{1}\right)<S c\left(\mathfrak{R}_{2}\right)$, then $\mathfrak{R}_{1}<\mathfrak{R}_{2}$;

. If $\operatorname{Sc}\left(\mathfrak{R}_{1}\right)=\operatorname{Sc}\left(\mathfrak{R}_{2}\right)$, then $\mathfrak{R}_{1}=\mathfrak{R}_{2}$.

Definition 9 Let $\mathfrak{R}_{1}=\left\{\left\langle\left[s_{\mu_{1}^{-}}^{\prime}, \dot{s}_{\mu_{1}^{+}}\right],\left(\dot{s}_{\lambda_{11}}^{\prime}, \dot{s}_{\lambda_{12}}, \ldots, \dot{s}_{\lambda_{1 p}}\right)\right\rangle\right.$, $\left.\left\langle\left[\dot{s}_{v_{1}^{-}}^{\prime}, \dot{s}_{v_{1}^{+}}\right],\left(\dot{s}_{\lambda_{11}}^{\prime}, \dot{s}_{\lambda_{12}}, \ldots, s_{\lambda_{1 p}}\right)\right\rangle\right\} \quad$ and $\quad \mathfrak{R}_{2}=\left\{\left\langle\left[s_{\mu_{2}^{-}}^{\prime}, s_{\mu_{2}^{+}}\right]\right.\right.$, $\left.\left.\left(\dot{s}_{\lambda_{21}}^{\prime}, \dot{s}_{\lambda_{22}}, \ldots, \dot{s}_{\lambda_{2 p}}\right)\right\rangle,\left\langle\left[\dot{s}_{v_{2}^{-}}^{\prime}, \dot{s}_{v_{2}^{+}}\right],\left(\dot{s}_{\lambda_{21}}^{\prime}, \dot{s}_{\lambda_{22}}, \ldots, \dot{s}_{\lambda_{2 p}}\right)\right\rangle\right\}$ be the two linguistic intuitionistic cubic hesitant variables in the CLTS $S^{*}=\left\{s_{\psi}^{\prime} \mid \dot{s}_{0} \leq \dot{s}_{\psi} \leq \dot{s}_{g}, \psi \in[0, \ell]\right.$ and $\delta>0$.. Then, the operational laws are defined as:

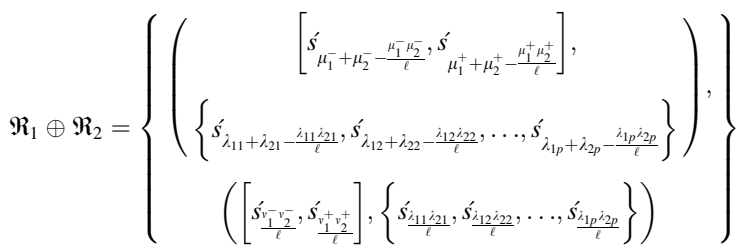

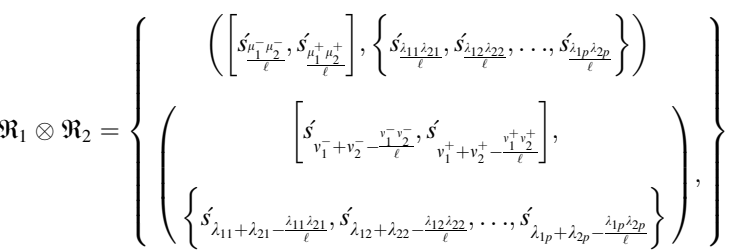

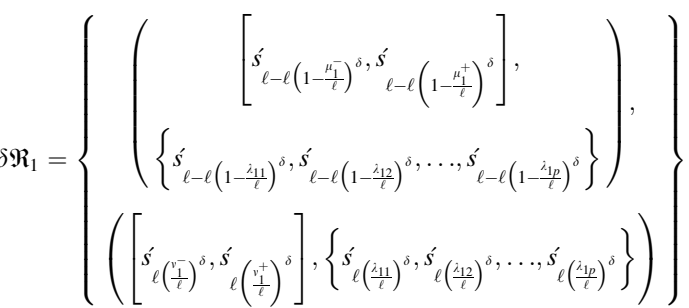

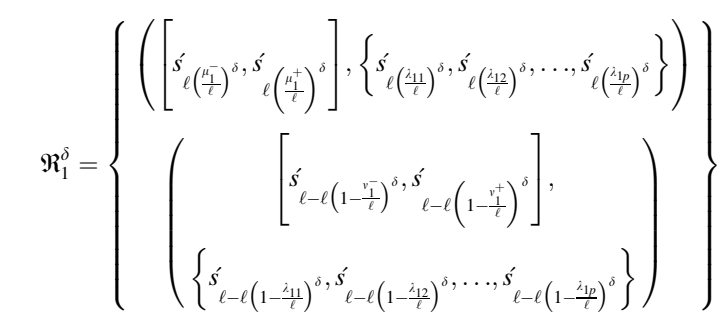




\section{Aggregation operators of linguistic intuitionistic cubic hesitant variables}

In this section, we developed some weighted averaging and geometric aggregation operators on linguistic intuitionistic cubic hesitant variables and discussed their basic properties.

\subsection{Weighted averaging aggregation operator of linguistic intuitionistic cubic hesitant variables}

Definition 10 Let $\mathfrak{R}_{\jmath}=\left\{\left\langle\left[s_{\mu_{\jmath}^{-}}, s_{\mu_{j}^{+}}\right],\left(s_{\lambda_{11}}, s_{\lambda_{\jmath 2}}, \ldots, s_{\lambda_{j p}}\right)\right\rangle\right.$, $\left.\left\langle\left[s_{v_{j}^{-}}^{\prime}, s_{v_{j}^{+}}\right]\left(s_{\lambda_{11}}, s_{\lambda_{j 2}}, \ldots, s_{\lambda_{j p}}\right)\right\rangle\right\}$ be the set of LICHVs in the CLTS $s^{*}=\left\{s_{\psi} \mid s_{0} \leq s_{\psi} \leq s_{g}, \psi \in[0, \ell]\right.$, with the vector $\Theta_{\jmath} \in[0,1]$ for $\sum_{\jmath=1}^{n} \Theta_{\jmath}=1$. Then, the corresponding WA operator of the LICHVs is defined as:

$\operatorname{LICHVWA}\left(\mathfrak{R}_{1}, \ldots, \mathfrak{R}_{3}\right)=\sum_{\jmath=1}^{n} \Theta_{\jmath} \Re_{\jmath}$.

Theorem 1 Let $\mathfrak{R}_{\jmath}=\left\{\left\langle\left[s_{\mu_{\jmath}^{-}}, s_{\mu_{\jmath}^{+}}\right],\left(s_{\lambda_{11}}, s_{\lambda_{\jmath 2}}, \ldots, s_{\lambda_{j p}}\right)\right\rangle\right.$, $\left.\left\langle\left[s_{v_{j}^{-}}^{\prime}, s_{v_{j}^{+}}\right],\left(s_{\lambda_{\lambda_{1}}}, s_{\lambda_{j 2}}, \ldots, s_{\lambda_{j p}}\right)\right\rangle\right\}$ be the set of LICHVs in the CLTS $S^{*}=\left\{s_{\psi} \mid \dot{s}_{0} \leq \dot{s}_{\psi} \leq \dot{s}_{g}, \psi \in[0, \ell]\right.$, with the vector $\Theta_{\jmath} \in[0,1]$ for $\sum_{\jmath=1}^{n} \Theta_{\jmath}=1$. Then, the aggregation result of Eq. (7) remains the LICHV, which is obtained by utilizing the following aggregation operation:

$$
\begin{aligned}
& \operatorname{LICHVWA}\left(\mathfrak{R}_{1}, \ldots, \mathfrak{R}_{3}\right)=\sum_{j=1}^{n} \Theta_{\jmath} \Re_{j}
\end{aligned}
$$

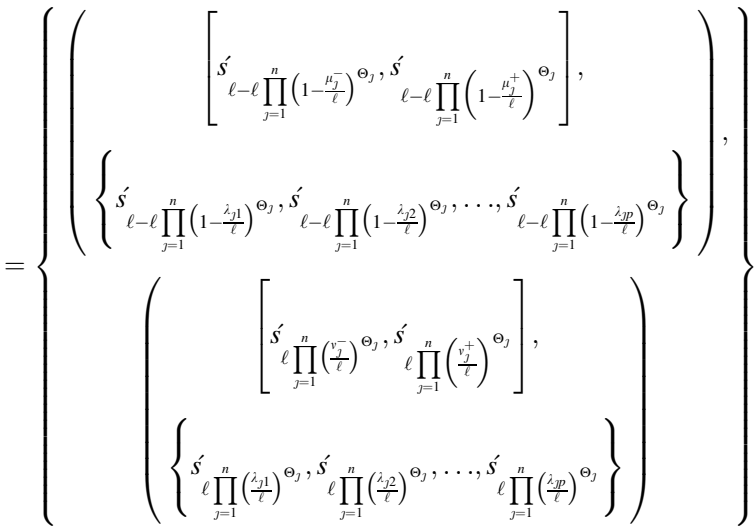

Proof (1). Put $n=2$, in operational law (3) of Definition 9 , we obtain

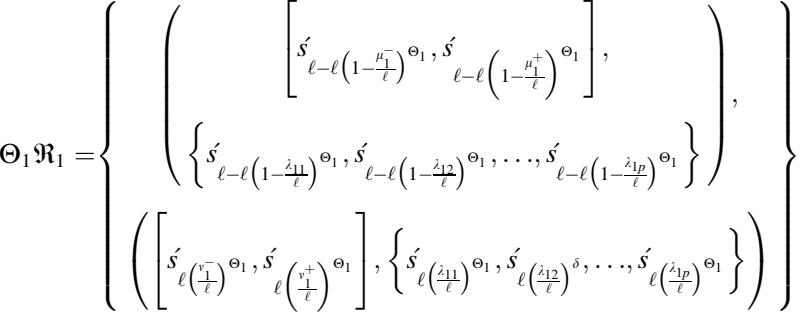

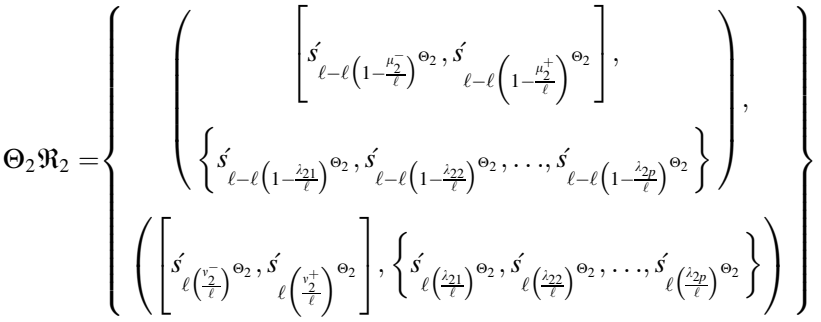

Using the operational law (1), the weighted aggregation result is obtain as:

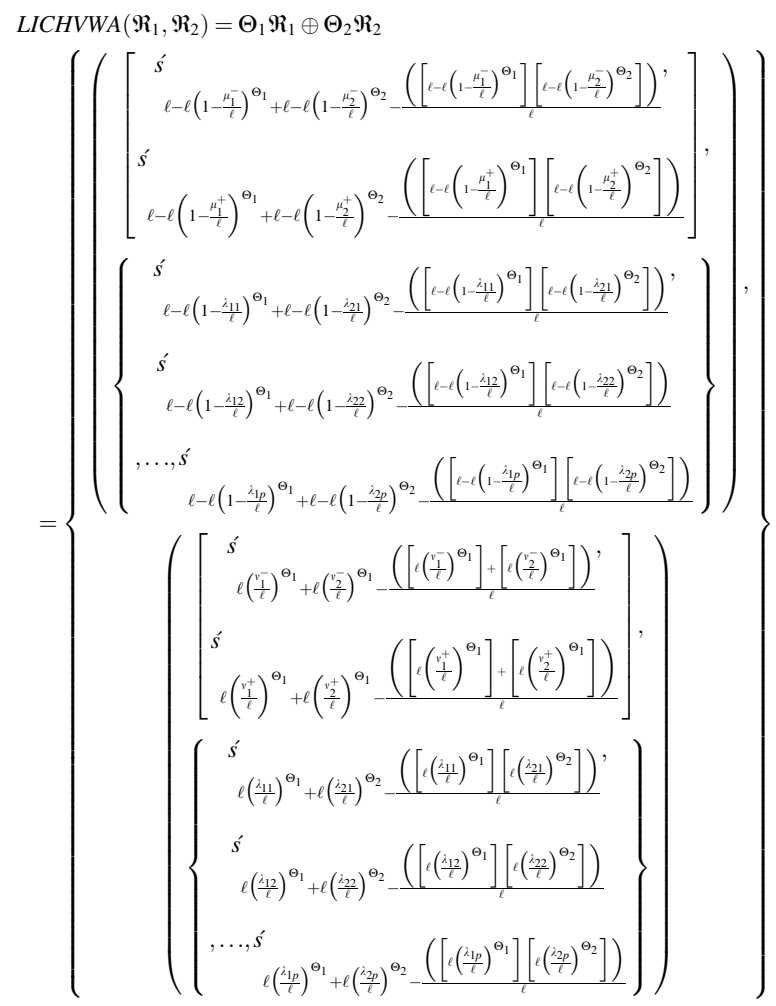




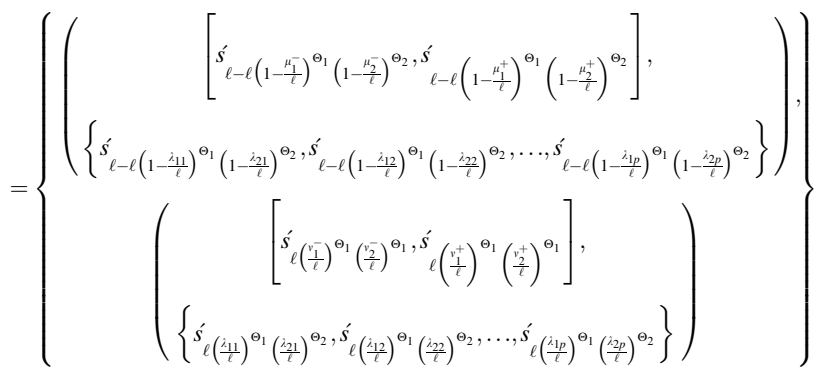

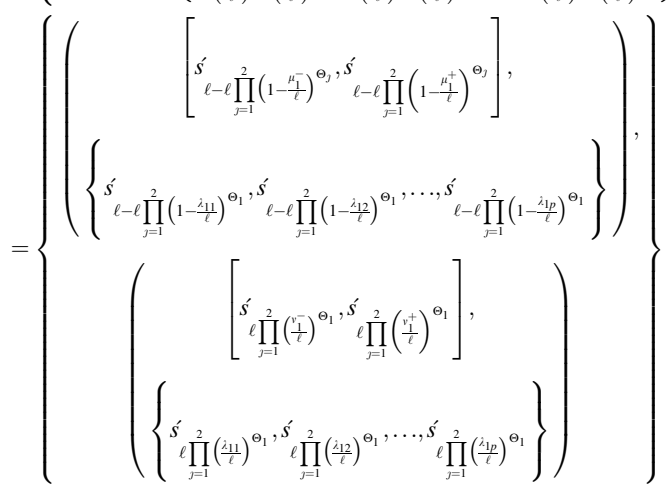

(2). Put $n=\kappa$, then the aggregation result of LICHVs based on Eq. (8) can be expressed as

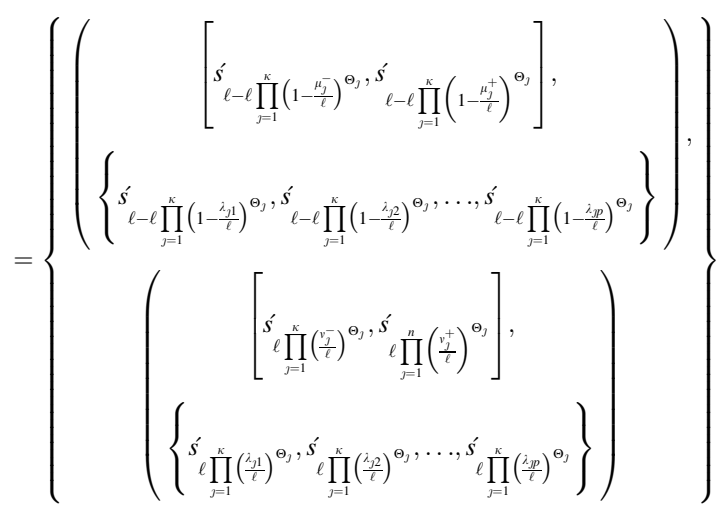

(3). Put $n=3$, then using Eqs. (A) and (B), the aggregation result of the LICHVs is given by

$$
\begin{aligned}
& \operatorname{LICHVWA}\left(\mathfrak{R}_{1}, \ldots, \Re_{\kappa}\right)=\sum_{\jmath=1}^{\kappa} \Theta_{\jmath} \Re_{\jmath} \oplus \boldsymbol{\Theta}_{\kappa+1} \Re_{\kappa+1}
\end{aligned}
$$

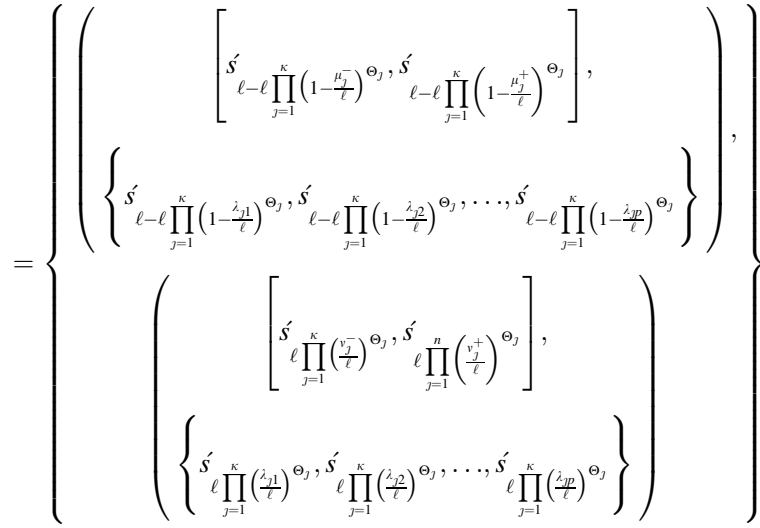

$$
\begin{aligned}
& +\Theta_{\kappa+1} \Re_{\kappa+1}
\end{aligned}
$$

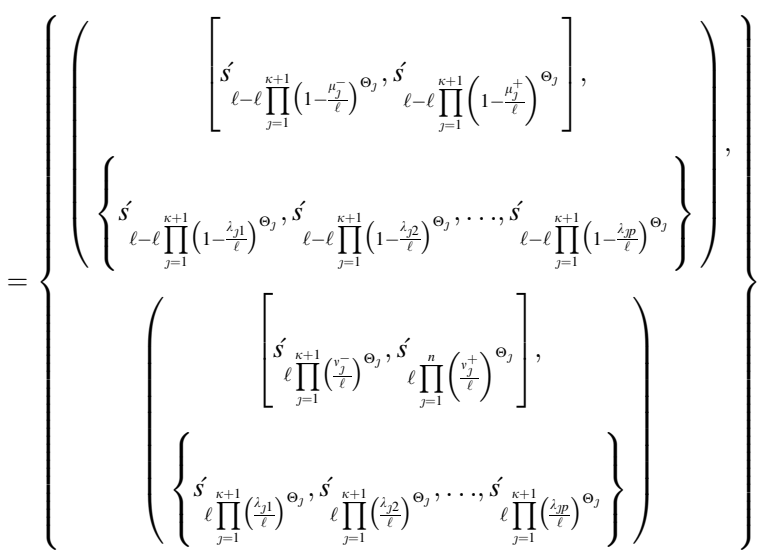

Hence, Eq. (8) is true for any $n$.

Theorem 2 Let $\quad \mathfrak{R}_{\jmath}=\left\{\left\langle\left[\dot{s}_{\mu_{j}^{-}}^{\prime}, s_{\mu_{\jmath}^{+}}\right],\left(s_{\lambda_{j 1}}, s_{\lambda_{j 2}}, \ldots, s_{\lambda_{j p}}\right)\right\rangle\right.$, $\left.\left\langle\left[s_{v_{j}^{-}}, s_{v_{j}^{+}}^{\prime}\right],\left(s_{\lambda_{11}}^{\prime}, s_{\lambda_{1,2}}, \ldots, s_{\lambda_{j p}}\right)\right\rangle\right\}$ be the set of LICHVs in the CLTS $S^{*}=\left\{s_{\psi} \mid \dot{s}_{0} \leq \dot{s}_{\psi} \leq \dot{s}_{g}, \psi \in[0, \ell]\right.$. Then, the WA operator of the LICHVWA $\left(\mathfrak{R}_{1}, \ldots, \mathfrak{R}_{n}\right)$ satisfies the following properties:

(1). (Idempotency): If $\mathfrak{R}_{\jmath}=\mathfrak{R}$, then there exist

$$
\operatorname{LICHVWA}\left(\mathfrak{R}_{1}, \ldots, \mathfrak{R}_{n}\right)=\mathfrak{R}_{\text {J }}
$$

Proof Since $\mathfrak{R}_{\jmath}=\mathfrak{R}(\jmath=1, \ldots, n)$, the WA aggregation result of LICHVs can be written as 


$$
\begin{aligned}
& \operatorname{LICHVWA}\left(\mathfrak{R}_{1}, \ldots, \mathfrak{R}_{n}\right)=\sum_{\jmath=1}^{n} \Theta_{\jmath} \Re_{\jmath}
\end{aligned}
$$

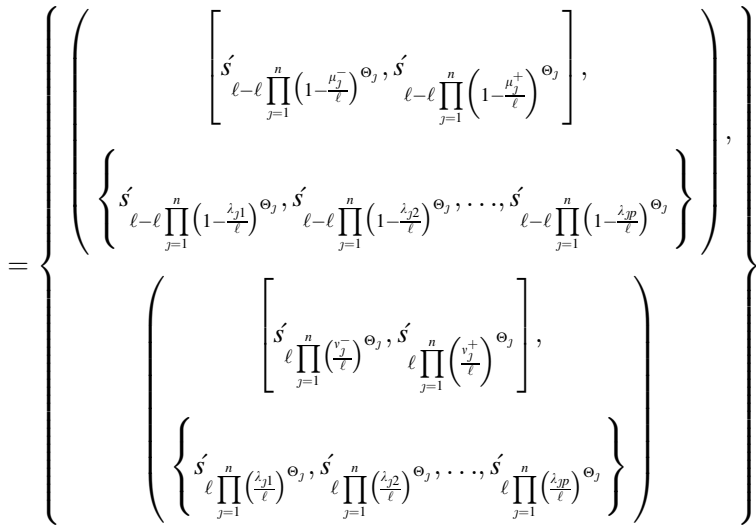

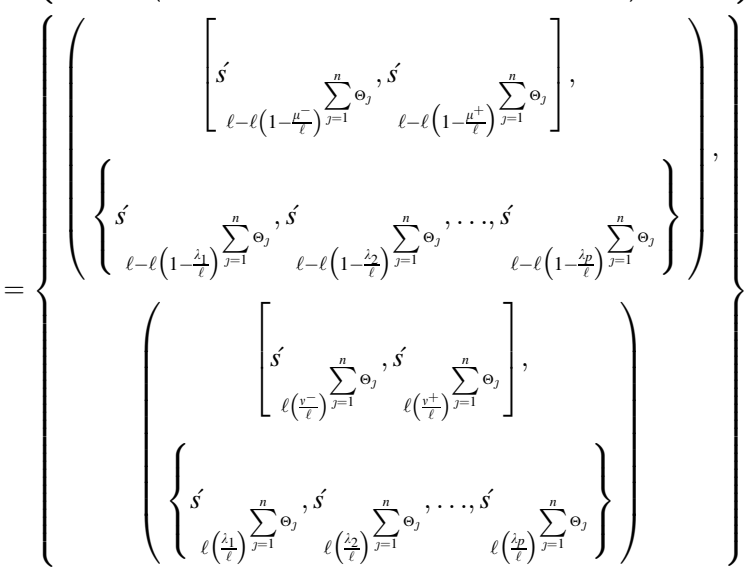

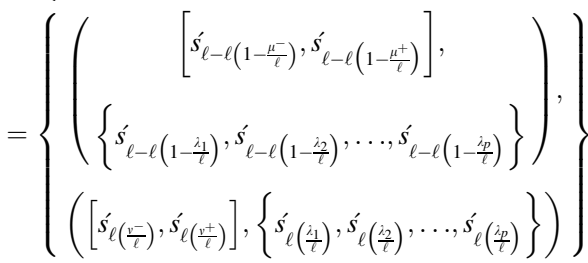

$$
\begin{aligned}
& =\left\{\left\langle\left[s_{\mu^{-}}, \dot{s}_{\mu^{+}}\right],\left(s_{\lambda_{1}}^{\prime}, s_{\lambda_{2}}^{\prime}, \ldots, s_{\lambda_{p}}^{\prime}\right)\right\rangle,\left\langle\left[s_{v^{-}}^{\prime}, s_{v^{+}}\right],\left(s_{\lambda_{1}}^{\prime}, s_{\lambda_{2}}^{\prime}, \ldots, s_{\lambda_{p}}\right)\right\rangle\right\} \\
& =\mathfrak{R}
\end{aligned}
$$

Theorem 3 (2). (Boundedness): If $\mathfrak{R}^{+}=$ $\left(\begin{array}{c}{\left[\max _{J}\left(s_{\mu_{J}^{\prime}}\right), \max _{J}\left(s_{\mu_{J}^{+}}\right)\right],\left[\min _{J}\left(s_{v_{J}^{-}}\right), \min _{J}\left(s_{v_{j}^{+}}\right)\right],} \\ \left\{\left[\max _{J}\left(s_{\lambda_{j 1}}\right), \max _{J}\left(s_{\lambda_{j 2}}\right), \ldots, \max _{J}\left(s_{\lambda_{j p}}\right)\right]\right\}\end{array}\right)$ and $\mathfrak{R}^{-}=\left(\begin{array}{c}{\left[\min _{j}\left(s_{\mu_{j}^{-}}\right), \min _{j}\left(\dot{s}_{\mu_{j}^{+}}\right)\right],\left[\max _{j}\left(s_{v_{j}^{\prime}}\right), \max _{j}\left(\dot{s}_{v_{j}^{+}}\right)\right],} \\ \left\{\left[\min _{j}\left(s_{\lambda_{j 1}}\right), \min _{j}\left(s_{\lambda_{j 2}}^{\prime}\right), \ldots, \min _{j}\left(\dot{s}_{\lambda_{j p}}\right)\right]\right\}\end{array}\right)(J=1, \ldots, n)$ are the maximum and minimum LICHVs, respectively, then,
$\mathfrak{R}^{-} \leq \operatorname{LICHVWA}\left(\mathfrak{R}_{1}, \ldots, \mathfrak{R}_{n}\right) \leq \mathfrak{R}^{+}$

Proof Since the minimum of LICHVs is $\mathfrak{R}^{-}$and the maximum is $\mathfrak{R}^{+}$, there exist $\mathfrak{R}^{-} \leq \mathfrak{R}_{\jmath} \leq \mathfrak{R}^{+}$. Thus, there are $\sum_{\jmath=1}^{n} \Theta_{\jmath} \mathfrak{R}^{-} \leq \sum_{\jmath=1}^{n} \Theta_{\jmath} \mathfrak{R}_{\jmath} \leq \sum_{\jmath=1}^{n} \Theta_{\jmath} \mathfrak{R}^{+}$. Based on the above property (1), there is $\sum_{\jmath=1}^{n} \Theta_{\jmath} \mathfrak{R}^{-}=\mathfrak{R}^{-}$and $\sum_{\jmath=1}^{n} \Theta_{\jmath} \mathfrak{R}^{+}=\mathfrak{R}^{+} . \quad$ Hence, $\quad \mathfrak{R}^{-} \leq \operatorname{LICHVWA}\left(\mathfrak{R}_{1}, \ldots\right.$, $\left.\mathfrak{R}_{n}\right) \leq \mathfrak{R}^{+}$.

Theorem 4 (3). (Monotonicity): If $\mathfrak{R}_{\jmath} \leq \mathfrak{R}^{*}$, then there exist

$\operatorname{LICHVWA}\left(\mathfrak{R}_{1}, \ldots, \mathfrak{R}_{n}\right) \leq \operatorname{LICHVWA}\left(\mathfrak{R}_{1}^{*}, \ldots, \mathfrak{R}_{n}^{*}\right)$

Proof Since $\mathfrak{R}_{\jmath} \leq \mathfrak{R}^{*}$ for $\jmath=1, \ldots, n$, there exists $\sum_{\jmath=1}^{n} \Theta_{\jmath} \mathfrak{R}_{\jmath} \leq \sum_{\jmath=1}^{n} \Theta_{\jmath} \mathfrak{R}_{\jmath}^{*}$. Hence,

$\operatorname{LICVWA}\left(\mathfrak{R}_{1}, \ldots, \mathfrak{R}_{n}\right) \leq \operatorname{LICVWA}\left(\mathfrak{R}_{1}^{*}, \ldots, \mathfrak{R}^{*}\right)$.

proved.

\subsection{Weighted geometric aggregation operator of linguistic intuitionistic cubic Hesitant variables}

Definition 11 Let $\mathfrak{R}_{\jmath}=\left\{\left\langle\left[s_{\mu_{\jmath}^{-}}, s_{\mu_{\jmath}^{+}}\right],\left(s_{\lambda_{\jmath 1}^{\prime}}, s_{\lambda_{j 2}}, \ldots, s_{\lambda_{j p}}\right)\right\rangle\right.$, $\left.\left\langle\left[s_{v_{j}^{-}}, s_{v_{j}^{+}}\right]\left(s_{\lambda_{j 1}^{\prime}}^{\prime}, s_{\lambda_{j 2}}, \ldots, s_{\lambda_{j p}}^{\prime}\right)\right\rangle\right\}$ be the set of LICHVs in the CLTS $S^{*}=\left\{s_{\psi} \mid s_{0} \leq \dot{s}_{\psi} \leq \dot{s}_{g}, \psi \in[0, \ell]\right.$, with the weight vector $\Theta_{\jmath} \in[0,1]$ for $\sum_{\jmath=1}^{n} \Theta_{\jmath}=1$. Then, the corresponding WG operator of the LICHVs is defined as:

$\operatorname{LICHVWG}\left(\mathfrak{R}_{1}, \ldots, \mathfrak{R}_{3}\right)=\sum_{\jmath=1}^{n}\left(\mathfrak{R}_{\jmath}\right)^{\Theta_{\jmath}}$.

Theorem 5 Let $\mathfrak{R}_{\jmath}=\left\{\left\langle\left[\dot{s}_{\mu_{J}^{-}}, s_{\mu_{j}^{+}}\right],\left(s_{\lambda_{j 1}}, s_{\lambda_{j 2}}, \ldots, s_{\lambda_{j p}}\right)\right\rangle\right.$, $\left.\left\langle\left[s_{v_{j}^{-}}, s_{v_{j}^{+}}\right],\left(s_{\lambda_{j 1}}^{\prime}, s_{\lambda_{j 2}}, \ldots, s_{\lambda_{\jmath p}}^{\prime}\right)\right\rangle\right\}$ be the set of LICHVs in the CLTS $S^{*}=\left\{\dot{s}_{\psi} \mid \dot{s}_{0} \leq s_{\psi} \leq \dot{s}_{g}, \psi \in[0, \ell]\right.$, with the vector $\Theta_{\jmath} \in[0,1]$ for $\sum_{\jmath=1}^{n} \Theta_{\jmath}=1$. Then, the aggregation result of Eq. (12), remains the LICHV, which is obtained by utilizing the following aggregation operation: 


$$
\begin{aligned}
& \operatorname{LICHVWG}\left(\Re_{1}, \ldots, \Re_{3}\right)=\sum_{\jmath=1}^{n}\left(\Re_{\jmath}\right)^{\Theta_{j}}
\end{aligned}
$$

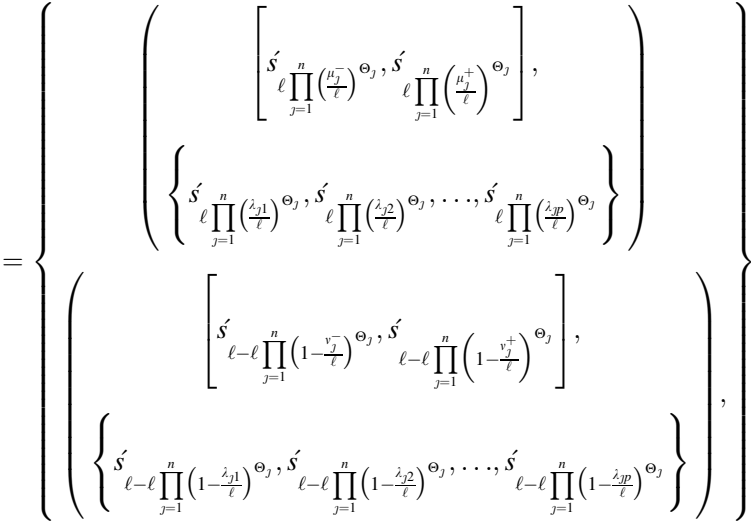

Proof The proof of this theorem is the same as the proof of Theorem 1, so we omitted the proof.

Theorem 6 Let $\mathfrak{R}_{\jmath}=\left\{\left\langle\left[s_{\mu_{\jmath}^{-}}^{\prime}, s_{\mu_{\jmath}^{+}}^{\prime}\right],\left(s_{\lambda_{j 1}}, s_{\lambda_{j 2}}, \ldots, s_{\lambda_{j p}}\right)\right\rangle\right.$, $\left.\left\langle\left[s_{v_{j}^{\prime}}^{\prime}, s_{v_{j}^{+}}^{\prime}\right],\left(s_{\lambda_{j 1}}, s_{\lambda_{j 2}}^{\prime}, \ldots, s_{\lambda_{\jmath p}}\right)\right\rangle\right\}$ be the set of LICHVs in the CLTS $S^{*}=\left\{\dot{s}_{\psi} \mid \dot{s}_{0} \leq \dot{s}_{\psi} \leq \dot{s}_{g}, \psi \in[0, \ell]\right.$. Then, the $W G$ operator of the LICHVWG( $\left.\mathfrak{R}_{1}, \ldots, \mathfrak{R}_{n}\right)$ satisfies the following properties:

(1). (Idempotency): If $\mathfrak{R}_{\jmath}=\mathfrak{R}$, then there exist

$$
\operatorname{LICHVWG}\left(\mathfrak{R}_{1}, \ldots, \mathfrak{R}_{n}\right)=\mathfrak{R}_{\jmath} .
$$

(2). (Boundedness): If

$$
\mathfrak{R}^{+}=\left(\begin{array}{c}
{\left[\max _{j}\left(s_{\mu_{j}^{-}}\right), \max _{j}\left(\dot{s}_{\mu_{j}^{+}}\right)\right],\left[\min _{j}\left(\dot{s}_{v_{j}^{-}}\right), \min _{j}\left(s_{v_{j}^{\prime}}\right)\right],} \\
\left\{\left[\max _{j}\left(\dot{s}_{\lambda_{j 1}}\right), \max _{j}\left(\dot{s}_{\lambda_{j 2}}\right), \ldots, \max _{j}\left(\dot{s}_{\lambda_{j p}}\right)\right]\right\}
\end{array}\right)
$$

and

$\mathfrak{R}^{-}=\left(\begin{array}{c}{\left[\min _{j}\left(s_{\mu_{j}^{-}}\right), \min _{j}\left(s_{\mu_{j}^{+}}\right)\right],\left[\max _{j}\left(s_{v_{j}^{-}}\right), \max _{j}\left(s_{v_{j}^{+}}\right)\right],} \\ \left\{\left[\min _{j}\left(s_{\lambda_{j 1}}\right), \min _{j}\left(s_{\lambda_{j 2}}^{\prime}\right), \ldots, \min _{j}\left(s_{\lambda_{j p}}\right)\right]\right\}\end{array}\right)$

$(\jmath=1, \ldots, n)$ are the maximum and minimum LICHVs, respectively, then,

$\mathfrak{R}^{-} \leq \operatorname{LICHVWG}\left(\mathfrak{R}_{1}, \ldots, \mathfrak{R}_{\kappa}\right) \leq \mathfrak{R}^{+}$.

(3). (Monotonicity): If $\mathfrak{R}_{\jmath} \leq \mathfrak{R}_{\jmath}^{*}$, then there exist

$\operatorname{LICHVWG}\left(\mathfrak{R}_{1}, \ldots, \mathfrak{R}_{\kappa}\right) \leq \operatorname{LICHVWG}\left(\mathfrak{R}_{1}^{*}, \ldots, \mathfrak{R}_{\kappa}^{*}\right)$.

Proof Since the proof of this theorem is the same as the proof of Theorem 2, it is omitted here.

\section{Algorithm of linguistic intuitionistic cubic hesitant variable for multi-criteria decision making problem}

In this section, we utilized the weighted averaging and geometric aggregation operators of linguistic intuitionistic cubic variables for multi-criteria decision making MCDM problem. Suppose that we have $n$ alternatives $\mathbb{Q}=$ $\left\{\mathbb{Q}_{1}, \ldots, \mathbb{Q}_{n}\right\}$ and $m$ criteria $\mathbb{N}=\left\{\mathbb{N}_{1}, \ldots, \mathbb{N}_{m}\right\}$ to be evaluated with associated weights that are $\Theta=$ $\left(\Theta_{1}, \ldots, \Theta_{n}\right)^{T}$ and $\Theta_{\jmath} \in[0,1], \sum_{\jmath=1}^{n} \Theta_{\jmath}=1$. To evaluate the performance of the alternative $\mathbb{Q}_{i}$ on the basis of criteria $\mathbb{N}_{\jmath}$, the decision makers provide the information about the alternative $\mathbb{Q}_{i}$ satisfying the criteria $\mathbb{N}_{j}$; they may assign an interval linguistic value to $\left(s_{\mu_{i j}}, s_{v_{i j}}\right)$ and a set of several possible linguistic values $\left(s_{\phi_{i j}}, s_{\chi_{i j}}\right)$, due to their hesitancy and indeterminacy from the presented CLTS $S^{*}=\left\{s_{\psi} \mid s_{0} \leq \dot{s}_{\psi} \leq s_{g}, \psi \in[0, \ell]\right.$, where $\ell$ is the even number. Thus, the combined information of $\left(s_{\mu_{i j}}, s_{v_{i j}}\right)$ and $\left(s_{\phi_{i j}}, s_{\chi_{i j}}\right)$ corresponding to the every criterion $\mathbb{N}_{\jmath}$ on each alternative $\mathbb{Q}_{i}$ can be presented as $\psi_{i j}=$ $\left\{\begin{array}{c}\left\langle\left[s_{\mu_{i j}^{-}}, s_{\mu_{i j}^{+}}\right],\left(s_{\lambda_{i j(1)}}, s_{\lambda_{i j(2)}}, \ldots, s_{\lambda_{i J(p)}}\right)\right\rangle, \\ \left\langle\left[s_{v_{i j}^{-}}, s_{v_{i j}^{+}}\right]\left(s_{\lambda_{i j(1)}}, s_{\lambda_{i j(2)}}, \ldots, s_{\lambda_{i j(p)}}\right)\right\rangle\end{array}\right\} \quad(i=1, \ldots n ;$ $\jmath=1, \ldots m)$. Hence, a LICHV decision matrix $M=$ $\left(\psi_{i j}\right)_{m \times n}$ can be constructed based on all the assessed LICHVs.

Thus, to aggregate the given data, we used the WA and WG operators and the linguistic score function of the LICHV to propose a MCDM algorithm under the LICHV setting, which has the following steps.

Step 1:The LCMNs of $\left(\dot{r}_{i 1}, \ldots, \dot{r}_{i n}\right)(i=1, \ldots, n)$ in $M=$ $\left(\psi_{i j}\right)_{m \times n}$ can be determined as $c_{i}$, where $r_{i j}$ is the numbers of LIVs in $\left(s_{\phi_{i j}}, s_{\varkappa_{i j}}\right)$ for $\psi_{i j}$. Based on the number of $\frac{c_{i}}{r_{i j}}$ in a $\operatorname{LICHV} \mathbb{Q}_{i \jmath}(i=1, \ldots n ; \jmath=1, \ldots m), \psi_{i \jmath}$ is extended to the following form: 
Granular Computing (2021) 6:691-703

699

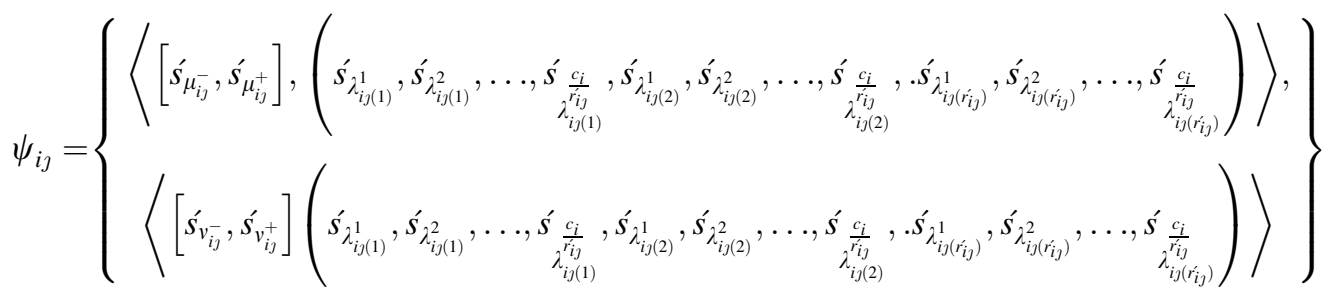

$$
\begin{aligned}
& =\left\{\left\langle\left[s_{\mu_{\jmath}^{-}}^{\prime}, s_{\mu_{j}^{+}}^{\prime}\right],\left(s_{\lambda_{j 1}^{\prime(1)}}, s_{\lambda_{j 2}^{\prime}}^{\prime 2)}, \ldots, s_{\lambda_{j p}^{\prime}\left(c_{i j}\right)}^{\prime}\right)\right\rangle,\left\langle\left[s_{v_{j}^{-}}^{\prime}, s_{v_{j}^{+}}\right],\left(s_{\lambda_{j 1}^{\prime(1)}}, s_{\lambda_{j 2}^{\prime}(2)}, \ldots, s_{\lambda_{j p}^{\left(c_{i}\right)}}^{\prime}\right)\right\rangle\right\}
\end{aligned}
$$

Table 1 The linguistic intuitionistic cubic hesitant variables decision matrix

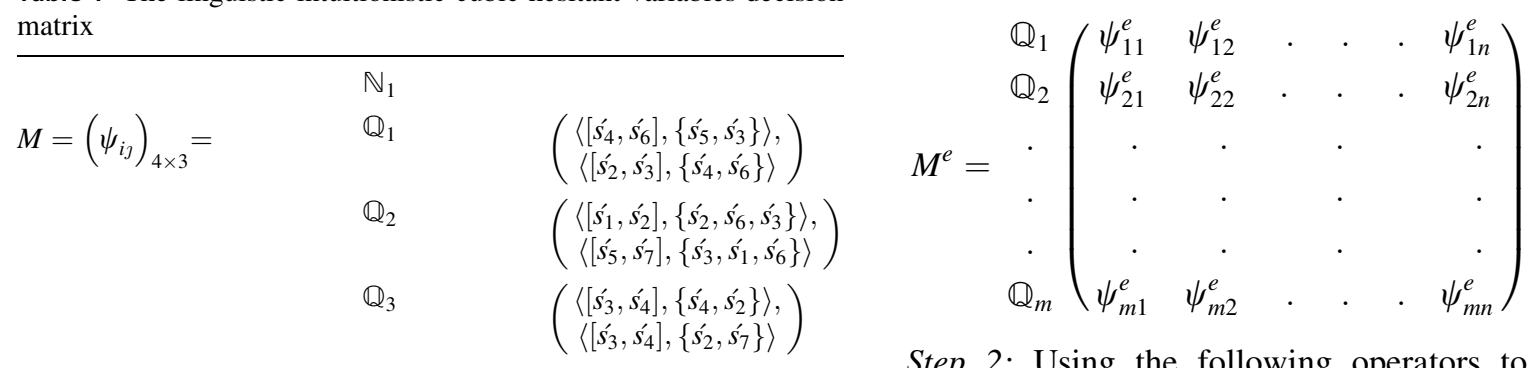

$$
\begin{aligned}
& \mathbb{Q}_{4} \quad\left(\begin{array}{c}
\left\langle\left[s_{2}, s_{3}^{\prime}\right],\left\{s_{3}^{\prime}, s_{7}^{\prime}\right\}\right\rangle, \\
\left\langle\left[s_{4}^{\prime}, s_{5}\right],\left\{s_{5}^{\prime}, s_{2}\right\}\right\rangle
\end{array}\right) \\
& \begin{array}{ll}
\mathbb{N}_{2} & \left(\begin{array}{l}
\left.\left\langle\dot{s}_{2}, s_{3}^{\prime}\right],\left\{s_{1}^{\prime}, s_{7}, s_{2}\right\}\right\rangle, \\
\mathbb{Q}_{1}
\end{array} \quad \begin{array}{l}
\left.\left\langle s_{4}, s_{6}^{\prime}\right],\left\{s_{5}, s_{3}, s_{1}^{\prime}\right\}\right\rangle
\end{array}\right)
\end{array} \\
& \mathbb{Q}_{2} \quad\left(\begin{array}{c}
\left\langle\left[s_{5}, s_{7}\right],\left\{s_{3}, s_{4}, s_{1}\right\}\right\rangle, \\
\left\langle\left[s_{1}, s_{2}\right],\left\{s_{2}, s_{7}, s_{3}\right\}\right\rangle
\end{array}\right) \\
& \mathbb{Q}_{3} \quad\left(\begin{array}{c}
\left\langle\left[s_{1}, s_{3}\right],\left\{s_{7}, s_{4}\right\}\right\rangle, \\
\left\langle\left[s_{2}, s_{5}\right],\left\{s_{1}, s_{6}\right\}\right\rangle
\end{array}\right) \\
& \mathbb{Q}_{4} \quad\left(\begin{array}{c}
\left\langle\left[s_{3}, \dot{s}_{4}\right],\left\{\dot{s}_{4}, s_{2}\right\}\right\rangle, \\
\left\langle\left[s_{2}, s_{3}^{\prime}\right],\left\{\dot{s}_{3}^{\prime}, s_{6}\right\}\right\rangle
\end{array}\right) \\
& \left.\begin{array}{ll}
\mathbb{N}_{3} & \left.\left(\begin{array}{l}
\left\langle\left[s_{3}^{\prime}, s_{4}\right],\left\{s_{3}^{\prime}, s_{6}\right\}\right\rangle, \\
\mathbb{Q}_{1}
\end{array} \quad s_{4}^{\prime}, s_{5}\right],\left\{s_{4}, s_{3}^{\prime}\right\}\right\rangle
\end{array}\right) \\
& \mathbb{Q}_{2} \quad\left(\begin{array}{l}
\left\langle\left[s_{3}, s_{5}\right],\left\{s_{4}^{\prime}, s_{6}\right\}\right\rangle, \\
\left\langle\left[s_{3}, s_{4}^{\prime}\right],\left\{s_{2}, s_{3}\right\}\right\rangle
\end{array}\right) \\
& \mathbb{Q}_{3} \quad\left(\begin{array}{c}
\left\langle\left[s_{2}, s_{5}\right],\left\{s_{1}, s_{7}, s_{2}\right\}\right\rangle, \\
\left\langle\left[s_{4}^{\prime}, s_{6}\right],\left\{s_{3}, s_{1}, s_{4}\right\}\right\rangle
\end{array}\right) \\
& \mathbb{Q}_{4} \quad\left(\begin{array}{c}
\left\langle\left[s_{5}^{\prime}, s_{7}\right],\left\{s_{2}^{\prime}, s_{3}, s_{1}\right\}\right\rangle, \\
\left\langle\left[s_{1}^{\prime}, s_{2}^{\prime}\right],\left\{s_{6}^{\prime}, s_{2}^{\prime}, s_{5}\right\}\right\rangle
\end{array}\right)
\end{aligned}
$$

Thus, we have the following extended matrix:

Springer 
Table 2 The extended linguistic intuitionistic cubic hesitant variables decision matrix

\begin{tabular}{|c|c|c|}
\hline \multirow{15}{*}{$M=\left(\psi_{i \jmath}\right)_{4 \times 3}=$} & $\mathbb{N}_{1}$ & \\
\hline & $\mathbb{Q}_{1}$ & $\left(\begin{array}{c}\left\langle\left[s_{4}^{\prime}, s_{6}^{\prime}\right],\left\{s_{5}^{\prime}, s_{5}, s_{5}, s_{3}^{\prime}, s^{\prime}, s_{3}\right\}\right\rangle, \\
\left\langle\left[s_{2}^{\prime}, s_{3}\right],\left\{s_{4}^{\prime}, s_{4}^{\prime}, s_{4}^{\prime}, s_{6}^{\prime}, s_{6}, s_{6}^{\prime}\right\}\right\rangle\end{array}\right)$ \\
\hline & $\mathbb{Q}_{2}$ & 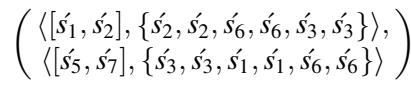 \\
\hline & $\mathbb{Q}_{3}$ & $\left(\begin{array}{c}\left\langle\left[s_{3}^{\prime}, s_{4}^{\prime}\right],\left\{s_{4}^{\prime}, s_{4}^{\prime}, s_{4}^{\prime}, s_{2}^{\prime}, s_{2}^{\prime}, s_{2}^{\prime}\right\}\right\rangle, \\
\left\langle\left[s_{3}^{\prime}, s_{4}^{\prime}\right],\left\{s_{2}^{\prime}, s_{2}^{\prime}, s_{2}, s_{7}^{\prime}, s_{7}^{\prime}, s_{7}^{\prime}\right\}\right\rangle\end{array}\right)$ \\
\hline & $\mathbb{Q}_{4}$ & $\left(\begin{array}{c}\left\langle\left[s_{2}^{\prime}, s_{3}\right],\left\{s_{3}^{\prime}, s_{3}, s_{3}, s_{7}, s_{7}, s_{7}\right\}\right\rangle, \\
\left\langle\left[s_{4}^{\prime}, s_{5}^{\prime}\right],\left\{s_{5}, s_{5}, s_{5}, s_{2}^{\prime}, s_{2}, s_{2}^{\prime}\right\}\right\rangle\end{array}\right)$ \\
\hline & $\mathbb{N}_{1}$ & \\
\hline & $\mathbb{Q}_{1}$ & $\left(\begin{array}{c}\left\langle\left[s_{2}^{\prime}, s_{3}\right],\left\{s_{1}^{\prime}, s_{1}^{\prime}, s_{7}^{\prime}, s_{7}, s_{2}, s_{2}\right\}\right\rangle, \\
\left\langle\left[s_{4}^{\prime}, s_{6}^{\prime}\right],\left\{s_{5}^{\prime}, s_{5}, s_{3}^{\prime}, s_{3}^{\prime}, s_{1}^{\prime}, s_{1}^{\prime}\right\}\right\rangle\end{array}\right)$ \\
\hline & $\mathbb{Q}_{2}$ & $\left(\begin{array}{c}\left\langle\left[s_{5}^{\prime}, s_{7}^{\prime}\right],\left\{s_{3}^{\prime}, s_{3}, s_{4}^{\prime}, s_{4}^{\prime}, s_{1}^{\prime}, s_{1}^{\prime}\right\}\right\rangle, \\
\left\langle\left[s_{1}^{\prime}, s_{2}^{\prime}\right],\left\{s_{2}^{\prime}, s_{2}^{\prime}, s_{7}, s_{7}^{\prime}, s_{3}^{\prime}, s_{3}^{\prime}\right\}\right\rangle\end{array}\right)$ \\
\hline & $\mathbb{Q}_{3}$ & 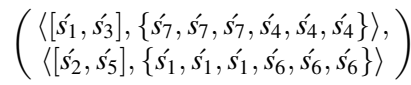 \\
\hline & $\mathbb{Q}_{4}$ & $\left(\begin{array}{c}\left\langle\left[s_{3}^{\prime}, s_{4}^{\prime}\right],\left\{s_{4}^{\prime}, s_{4}^{\prime}, s_{4}^{\prime}, s_{2}^{\prime}, s_{2}^{\prime}, s_{2}^{\prime}\right\}\right\rangle, \\
\left\langle\left[s_{2}^{\prime}, s_{3}^{\prime}\right],\left\{s_{3}^{\prime}, s_{3}^{\prime}, s_{3}^{\prime}, s_{6}^{\prime}, s_{6}^{\prime}, s_{6}^{\prime}\right\}\right\rangle\end{array}\right)$ \\
\hline & $\mathbb{N}_{1}$ & \\
\hline & $\mathbb{Q}_{1}$ & $\left(\begin{array}{c}\left\langle\left[s_{3}^{\prime}, s_{4}^{\prime}\right],\left\{s_{3}^{\prime}, s_{3}^{\prime}, s_{3}, s_{6}^{\prime}, s_{6}^{\prime}, s_{6}^{\prime}\right\}\right\rangle, \\
\left\langle\left[s_{4}^{\prime}, s_{5}\right],\left\{s_{4}^{\prime}, s_{4}^{\prime}, s_{4}, s_{3}^{\prime}, s_{3}^{\prime}, s_{3}^{\prime}\right\}\right\rangle\end{array}\right)$ \\
\hline & $\mathbb{Q}_{2}$ & $\left(\begin{array}{c}\left\langle\left[s_{3}^{\prime}, s_{5}^{\prime}\right],\left\{s_{4}^{\prime}, s_{4}^{\prime}, s_{4}^{\prime}, s_{6}^{\prime}, s_{6}^{\prime}, s_{6}^{\prime}\right\}\right\rangle, \\
\left\langle\left[s_{3}^{\prime}, s_{4}^{\prime}\right],\left\{s_{2}^{\prime}, s_{2}^{\prime}, s_{2}, s_{3}^{\prime}, s_{3}^{\prime}, s_{3}^{\prime}\right\}\right\rangle\end{array}\right)$ \\
\hline & $\mathbb{Q}_{3}$ & $\left(\begin{array}{c}\left\langle\left[s_{2}^{\prime}, s_{5}\right],\left\{s_{1}^{\prime}, s_{1}^{\prime}, s_{7}^{\prime}, s_{7}, s_{2}, s_{2}\right\}\right\rangle, \\
\left\langle\left[s_{4}^{\prime}, s_{6}^{\prime}\right],\left\{s_{3}, s_{3}^{\prime}, s_{1}, s_{1}^{\prime}, s_{4}^{\prime}, s_{4}^{\prime}\right\}\right\rangle\end{array}\right)$ \\
\hline & $\mathbb{Q}_{4}$ & $\left(\begin{array}{c}\left\langle\left[s_{5}^{\prime}, s_{7}^{\prime}\right],\left\{s_{2}^{\prime}, s_{2}^{\prime}, s_{3}^{\prime}, s_{3}, s_{1}^{\prime}, s_{1}^{\prime}\right\}\right\rangle, \\
\left\langle\left[s_{1}^{\prime}, s_{2}^{\prime}\right],\left\{s_{6}^{\prime}, s_{6}^{\prime}, s_{2}, s_{2}^{\prime}, s_{5}^{\prime}, s_{5}^{\prime}\right\}\right\rangle\end{array}\right)$ \\
\hline
\end{tabular}

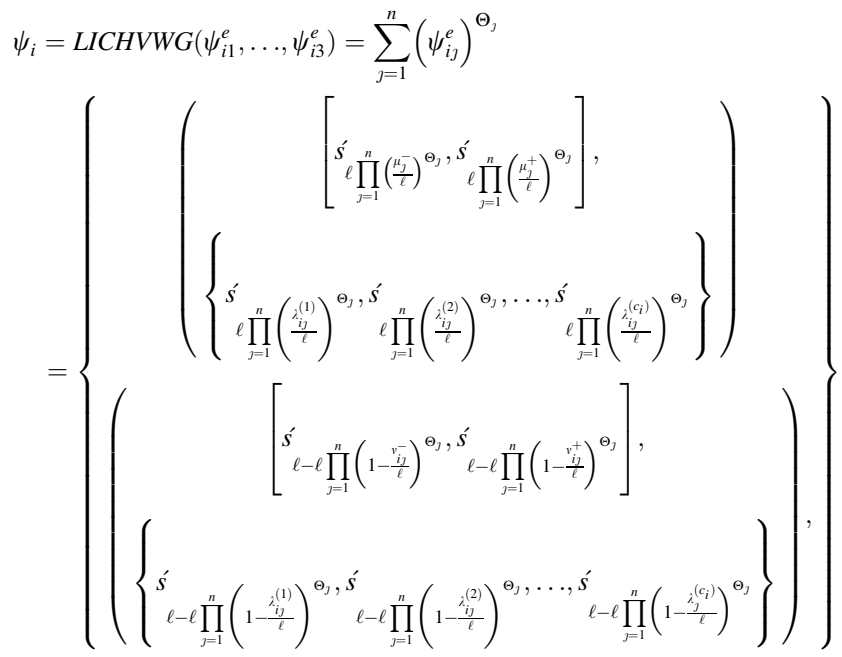

Step 3: To find the best alternative, find the linguistic score values of each alternative by using Eq. (6).
Step 4: To give ranking to the alternatives, write the score values of the alternatives in the ascending order and choose the biggest one(s).

\section{Example}

A fire and explosion incident occurred on August 12, 2015, in a chemical factory. In this incident, 75 persons were dead and more than 500 were injured. Due to this incident, of about 4745 million Dollars economy lost. This incident causes huge economic losses, many casualties effected the environment very badly. A number of buildings and vehicles were damaged. The environment of these areas was heavily polluted. To avoid additional losses, emergency response system should be provided. Four things are necessary to arrange the emergency response system.

(1) $\mathbb{N}_{1}$ : People effected

(2) $\mathbb{N}_{2}:$ Environmental effect

(3) $\mathbb{N}_{3}$ : Social impact.

Decision makers apply the LICHVs to evaluate the four alternatives under the three criteria with the weighting vector $w=(0.20,0.38,0.42)^{T}$.

Subsequently, the LICHVs evaluation matrix $\psi=\psi_{\imath j}$ is obtained as shown in Table 1

Step 1: The LCMNs of $\left(\dot{r}_{i 1}, \ldots, \dot{r}_{\text {in }}\right)(i=1, \ldots, m)$ in $M=\left(\mathbb{Q}_{i j}\right)_{m \times n}$ can be determined as $c_{i}=6$ for $i=1, \ldots, 4$. Applying the LCMN method, the extended decision matrix can be obtained, as shown in Table 2:

Step 2: Using Eq. (8), to obtain the aggregated LICHV $\psi_{1}$ for $\mathbb{Q}_{1}$ as follows:

$\psi_{1}=\operatorname{LICHVWA}\left(\psi_{11}^{e}, \psi_{12}^{e}, \psi_{13}^{e}\right)=\sum_{\jmath=1}^{3} \Theta_{\jmath} \psi_{1_{j}}^{e}$

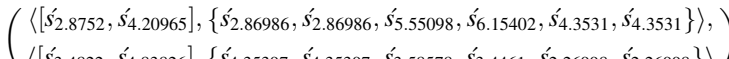

$\psi_{2}=\left(\begin{array}{l}\left\langle\left[s_{3.59554}, \dot{s}_{5.73002}\right],\left\{\dot{s}_{3.27824}^{\prime}, \dot{s}_{3.27824}, \dot{s}_{4.5178}, s_{5.39732}, \dot{s}_{4.13309}, \dot{s}_{4.13309}\right\}\right\rangle, \\ \left.\left\langle\dot{s}_{2.18869}, \dot{s}_{3.43777}\right],\left\{\dot{s}_{2.16894}, \dot{s}_{2.16894}, \dot{s}_{2.80266}, \dot{s}_{3.32298}, \dot{s}_{3.4461}, \dot{s}_{4.13309}\right\}\right\rangle\end{array}\right)$

$\psi_{3}=\left(\begin{array}{c}\left\langle\left[s_{1.86584}, s_{4.14156}\right],\left\{s_{5.01219}, s_{5.01219}, s_{6.68049}, s_{5.57667}, s_{2.85676}, s_{2.85676}\right\}\right\rangle, \\ \left\langle\left[s_{2.90189}, s_{5.16231}\right],\left\{s_{1.82223}, s_{1.82224}, s_{1.14871}, s_{2.91553}, s_{5.21894}, s_{5.21894}\right\}\right\rangle\end{array}\right)$

$\psi_{4}=\left(\begin{array}{c}\left\langle\left[\dot{s}_{3.81563}, \dot{s}_{5.66344}\right],\left\{\dot{s}_{3.04093}, \dot{s}_{3.04093}, \dot{s}_{3.40649}, \dot{s}_{4.11613}, \dot{s}_{3.52659}, \dot{s}_{3.52659}\right\}\right\rangle, \\ \left\langle\left[\dot{s}_{1.71713}, \dot{s}_{2.80242}\right],\left\{\dot{s}_{4.44553}, \dot{s}_{4.44553}, \dot{s}_{2.80242}, \dot{s}_{3.03624}, \dot{s}_{4.4614}, \dot{s}_{4.4614}\right\}\right\rangle\end{array}\right)$

Step 3: Using Eq. (8), we find the linguistic score value of all the alternatives:

$$
\begin{aligned}
& S c\left(\mathbb{Q}_{1}\right)=s_{1.9301}, S c\left(\mathbb{Q}_{2}\right)=s_{1.8257}, S c\left(\mathbb{Q}_{3}\right)=s_{1.8395}, \\
& S c\left(\mathbb{Q}_{1}\right)=s_{1.17980}
\end{aligned}
$$

Step 4: Based on the linguistic score values, we give ranking to the alternatives, as follows:

$\mathbb{Q}_{1}>\mathbb{Q}_{3}>\mathbb{Q}_{2}>\mathbb{Q}_{4}$ 
Table 3 Comparison with the other methods

\begin{tabular}{llllll}
\hline Score values & $\mathbb{Q}_{1}$ & $\mathbb{Q}_{2}$ & $\mathbb{Q}_{3}$ & $\mathbb{Q}_{4}$ & Ranking \\
\hline Garg and Kumar (2019) & 4.8413 & 4.3551 & 4.6911 & 3.6823 & $\mathbb{Q}_{1}>\mathbb{Q}_{3}>\mathbb{Q}_{2}>\mathbb{Q}_{4}$ \\
& 4.3174 & 3.6942 & 3.5732 & 3.1341 & $\mathbb{Q}_{1}>\mathbb{Q}_{2}>\mathbb{Q}_{3}>\mathbb{Q}_{4}$ \\
Liu et al. (2017) & 5.6379 & 3.1933 & 4.3734 & 4.9381 & $\mathbb{Q}_{1}>\mathbb{Q}_{4}>\mathbb{Q}_{3}>\mathbb{Q}_{2}$ \\
Lu and Ye (2019) & 2.7471 & 1.9184 & 2.0421 & 2.5921 & $\mathbb{Q}_{1}>\mathbb{Q}_{4}>\mathbb{Q}_{3}>\mathbb{Q}_{2}$ \\
LICHVWA operator & $s_{1.9301}$ & $s_{1.8257}$ & $s_{1.8395}$ & $s_{1.17980}$ & $\mathbb{Q}_{1}>\mathbb{Q}_{3}>\mathbb{Q}_{2}>\mathbb{Q}_{4}$ \\
LICHVWG operator & $s_{1.8856}$ & $s_{1.8788}$ & $s_{1.8343}$ & $s_{1.17980}$ & $\mathbb{Q}_{1}>\mathbb{Q}_{3}>\mathbb{Q}_{2}>\mathbb{Q}_{4}$ \\
\hline
\end{tabular}

Now, we can use the developed MCDM method based on the WG operator of LICHVs for the example.

Step 1:This step is the same as the previous step 1 .

Step 2:Using Eq. (14), to obtain the aggregated LICHV of $\psi_{1}$ for $\mathbb{Q}_{1}$, as follows:

$\psi_{1}=L I C H V W G\left(\psi_{11}^{e}, \psi_{12}^{e}, \psi_{13}^{e}\right)=\sum_{j=1}^{3} \Theta_{\jmath} \psi_{1 j}^{e}$

$\left(\left\langle\left[\dot{s}_{2.72392}, \dot{s}_{3.88867}\right],\left\{\dot{s}_{2.18869}, \dot{s}_{2.18869}, \dot{s}_{4.58482}, \dot{s}_{5.53841}, \dot{s}_{3.44064}, \dot{s}_{3.44064}\right\}\right\rangle\right.$,

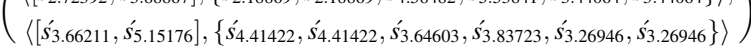

$\psi_{2}=\left(\begin{array}{c}\left\langle\left[\dot{s}_{2.92415}, \dot{s}_{4.73054}\right],\left\{\dot{s}_{3.1216}, \dot{s}_{3.1216}, \dot{s}_{4.33789}, \dot{s}_{5.14324}, \dot{s}_{2.64392}, \dot{s}_{2.64392}\right\}\right\rangle, \\ \left\langle\left[\dot{s}_{2.86986}, \dot{s}_{4.46359}\right],\left\{\dot{s}_{2.21484}, \dot{s}_{2.21484}, \dot{s}_{4.86785}, \dot{s}_{5.09874}, \dot{s}_{3.83723}, \dot{s}_{3.83723}\right\}\right\rangle\end{array}\right)$

$\psi_{3}=\left(\begin{array}{l}\left\langle\left[\dot{s}_{1.6667}, \dot{s}_{3.93809}\right],\left\{\dot{s}_{2.76407}, \dot{s}_{2.76407}, \dot{s}_{6.25879}, \dot{s}_{4.40484}, \dot{s}_{2.60268}, \dot{s}_{2.60268}\right\}\right\rangle, \\ \left\langle\left[\dot{s}_{3.12071}, \dot{s}_{5.3199}\right],\left\{\dot{s}_{2.10702}, \dot{s}_{2.10702}, \dot{s}_{1.21252}, \dot{s}_{5.05332}, \dot{s}_{5.67053}, \dot{s}_{5.67053}\right\}\right\rangle\end{array}\right)$

$\psi_{4}=\left(\begin{array}{c}\left\langle\left[\dot{s}_{3.42831}, \dot{s}_{4.77692}\right],\left\{\dot{s}_{2.82254}, \dot{s}_{2.82254}, \dot{s}_{3.34656}, \dot{s}_{3.04651}, \dot{s}_{1.92049}, \dot{s}_{1.92049}\right\}\right\rangle, \\ \left\langle\left[\dot{s}_{2.0973}, \dot{s}_{3.12633}\right],\left\{\dot{s}_{4.92769}, \dot{s}_{4.92769}, \dot{s}_{3.12633}, \dot{s}_{4.04774}, \dot{s}_{5.04599}, \dot{s}_{5.04599}\right\}\right\rangle\end{array}\right)$

Step 3:Using Eq. (6), we find the linguistic value of all the alternatives:

$S c\left(\mathbb{Q}_{1}\right)=\dot{s}_{1.8856}, S c\left(\mathbb{Q}_{2}\right)=\dot{s}_{1.8788}, \operatorname{Sc}\left(\mathbb{Q}_{3}\right)=s_{1.8343}$,

$\operatorname{Sc}\left(\mathbb{Q}_{1}\right)=s_{1.7884}$

Step 4:Based on the linguistic score values, we give ranking to the alternatives, as follows:

$\mathbb{Q}_{1}>\mathbb{Q}_{3}>\mathbb{Q}_{2}>\mathbb{Q}_{4}$

\section{Comparative study}

In this study, we compared our proposed advance developed aggregation operators to preexisting fuzzy aggregation operators and also the conclusion of our work is stated. Despite the fact that LIF set theory has an incredible effect in different fields, there are some realworld problems, which were not possible by LIFS and even not possible to be solved by IVLIFS. In LICHVs, each element consists of the linguistic membership grade and linguistic non-membership grade. If we consider the developed numerical problem in Section 6, as LICHV is the most advance structure, it is not possible for the existing aggregation operators to solve the data contained in the said problem, which shows the limited approach of the existing approaches. But if we consider any problem under the interval-valued fuzzy information, we can solve it easily by the LICHVs by converting the data from the interval valued to LICHVs, taking the values outside the interval in LICHVs equal to zero.

Now, we compare our approach with that of the Garg and Kumar (2019), Liu et al. (2017), and $\mathrm{Lu}$ and Ye (2019). In order to compare our developed method with the other methods above, each linguistic term has one positive and one negative grade. So, if we only consider the positive and negative grades, we neglect the cubic term, and then the LICHVs reduce to LIVIF numbers. We take $\Theta=$ $(0.3,0.2,0.1,0.4)^{T}$ as the attribute weight vector to facilitate the comparison. Based on given preferences and the information, we apply the existing approaches on the considered data and then the final score value of the alternatives $\mathbb{Q}_{l}(l=1, \ldots, 4)$ is given in Table 3. From Table 3, we observe that the best alternative in all approaches is $\mathbb{Q}_{1}$. However, there are some differences in the remaining alternatives, due to different evaluation. Thus, our proposed method is more better than the other existing methods.

\section{Conclusion}

In this study, we introduced the linguistic intuitionistic cubic hesitant variables, which expressed the hybrid form of interval/uncertain linguistic and hesitant linguistic information. We defined the score function for the comparison of linguistic intuitionistic cubic hesitant variables. Some linguistic intuitionistic cubic hesitant variables operational laws have been developed. Furthermore, we proposed the weighted average and weighted geometric aggregation operators utilizing the concept of LCMN extension method. We also discussed some of its properties like idempotency, boundary, and monotonicity. A multiple criteria decision making approach was developed, based on the WA and WG operators of the LICHVs to solve a MCDM problem under the LICHV information. To show the effectiveness of these operators, a numerical example has been presented which shows that the suggested operators deliver an alternative way to solve decision making 
process in a more actual way. Finally, we have provided some comparison with the existing operators to show the validity and effectiveness of the novel methodology.

In future work, we will further develop more aggregation operators under LICHV information, like Dombi aggregation operators, Himachar aggregation operators, Dombi Bonferroni mean operators, and some more. We will also expand TOPSIS, VIKOR, and few other methods under LICHV environment and will apply them to expand a number of strategies to resolve MCGDM problems, risk evaluation, fault diagnosis, and other domains under indeterminate conditions.

\section{Compliance with ethical standards}

Conflict of interest The authors declare that they have no conflict of interest.

Open Access This article is licensed under a Creative Commons Attribution 4.0 International License, which permits use, sharing, adaptation, distribution and reproduction in any medium or format, as long as you give appropriate credit to the original author(s) and the source, provide a link to the Creative Commons licence, and indicate if changes were made. The images or other third party material in this article are included in the article's Creative Commons licence, unless indicated otherwise in a credit line to the material. If material is not included in the article's Creative Commons licence and your intended use is not permitted by statutory regulation or exceeds the permitted use, you will need to obtain permission directly from the copyright holder. To view a copy of this licence, visit http://creativecommons. org/licenses/by/4.0/.

\section{References}

Atanasav K (1986) Intuitionistic fuzzy sets. Fuzzy Sets Syst 20(1):87-96

Bai SM, Chen SM (2006) Automatically constructing grade membership functions for students' evaluation for fuzzy grading systems. In: 2006 World Automation Congress, pp 1-6. IEEE

Bai SM, Chen SM (2008) Automatically constructing concept maps based on fuzzy rules for adapting learning systems. Expert Syst Appl 35(1-2):41-49

Beliakov G, Bustince H, Goswami D, Mukherjee U, Pal N (2011) On averaging operators for Atanassov's intuitionistic fuzzy sets. Inf Sci 181(6):1116-1124

Chen SM, Ke JS, Chang JF (1992) An inexact reasoning algorithm based on fuzzy rule matrix transformations. J Autom Reason 8(1):77-90

Chen SM (1996) A fuzzy reasoning approach for rule-based systems based on fuzzy logics. IEEE Trans Syst Man Cybern Part B (Cybernetics) 26(5):769-778

Chen SM, Tsai FM (2008) Generating fuzzy rules from training instances for fuzzy classification systems. Expert Syst Appl 35(3):611-621

Chen SM, Chu HP, Sheu TW (2012) TAIEX forecasting using fuzzy time series and automatically generated weights of multiple factors. IEEE Trans Syst Man Cybern Part A Syst Humans 42(6): 1485-1495
Chen SM, Manalu GMT, Pan JS, Liu HC (2013) Fuzzy forecasting based on two-factors second-order fuzzy-trend logical relationship groups and particle swarm optimization techniques. IEEE Trans Cybern 43(3):1102-1117

Chen SM, Chen SW (2014) Fuzzy forecasting based on two-factors second-order fuzzy-trend logical relationship groups and the probabilities of trends of fuzzy logical relationships. IEEE Trans Cybern 45(3):391-403

Chen SM, Huang MH (2014) Automatically generating the weather news summary based on fuzzy reasoning and ontology techniques. Inf Sci 279:746-763

Chen SM, Cheng SH, Lan TC (2016) A novel similarity measure between intuitionistic fuzzy sets based on the centroid points of transformed fuzzy numbers with applications to pattern recognition. Inf Sci 343:15-40

Fahmi A, Abdullah S, Amin F, Siddiqui N, Ali A (2017) Aggregation operators on triangular cubic fuzzy numbers and its application to multi-criteria decision making problems. J Intell Fuzzy Syst 33(6):3323-3337

Fahmi A, Abdullah S, Amin F, Ahmed R, Ali A (2018a) Triangular cubic linguistic hesitant fuzzy aggregation operators and their application in group decision making. J Intell Fuzzy Syst 3:1-15

Fahmi A, Abdullah S, Amin F, Ali A, Ahmad KW (2018b) Some geometric operators with triangular cubic linguistic hesitant fuzzy number and their application in group decision-making. J Intell Fuzzy Syst 3:1-15

Fahmi A, Abdullah S, Amin F, Ali A (2018c) Weighted average rating (war) method for solving group decision making problem using triangular cubic fuzzy hybrid aggregation (tcfha). Punjab Univ J Math 50(1):23-34

Fahmi A, Abdullah S, Amin F (2018d) Expected values of aggregation operators on cubic trapezoidal fuzzy number and its application to multi-criteria decision making problems. J New Theory 22:51-65

Fahmi A, Amin F, Abdullah S, Ali A (2018e) Cubic fuzzy Einstein aggregation operators and its application to decision-making. Int J Syst Sci 49(11):2385-2397

Fahmi A, Amin F, Abdullah S, Aslam M, Ul Amin N (2018f) Cubic Fuzzy multi-attribute group decision-making with an application to plant location selected based on a new extended Vikor method. J Intell Fuzzy Syst 3:1-14

Fahmi A, Abdullah S, Amin F, Khan MSA (2019) Trapezoidal cubic fuzzy number Einstein hybrid weighted averaging operators and its application to decision making. Soft Comput 23(14):5753-5783

Garg H (2017) Generalized Pythagorean fuzzy geometric aggregation operators using Einstein t-norm and t-conorm for multicriteria decision-making process. Int J Intell Syst 32(6):597-630

Gitinavard H, Mousavi SM, Vahdani B (2017) Soft computing-based new interval-valued hesitant fuzzy multi-criteria group assessment method with last aggregation to industrial decision problems. Soft Comput 21(12):3247-3265

Garg H, Kumar K (2019) Linguistic interval-valued Atanassov intuitionistic fuzzy sets and their applications to group decisionmaking problems. IEEE Trans Fuzzy Syst 3:10

Herrera F, Herrera-Viedma E (2000) Linguistic decision analysis: steps for solving decision problems under linguistic information. Fuzzy Sets Syst 115(1):67-82

Jun YB, Kim CS, Yang KO (2011) Annals of fuzzy mathematics and informatics. Cubic Sets 4:83-98

Kim SH, Ahn BS (1999) Interactive group decision making procedure under incomplete information. Eur J Oper Res 116(3):498-507

Kou G, Ergu D, Lin C, Chen Y (2016) Pairwise comparison matrix in multiple criteria decision making. Technol Econ Dev Econ 22(5):738-765 
Kaur G, Garg H (2018) Cubic intuitionistic fuzzy aggregation operators. Int J Uncertain Quantifi 8(5):10

Kaur G, Garg H (2018) Multi-attribute decision-making based on bonferroni mean operators under cubic intuitionistic fuzzy set environment. Entropy 20(1):65

Kaur G, Garg H (2019) Generalized cubic intuitionistic fuzzy aggregation operators using t-norm operations and their applications to group decision-making process. Arab J Sci Eng 44(3):2775-2794

Li DF (2010) Multiattribute decision maki8ng method based on generalized OWA operators with intuitionistic fuzzy sets. Expert Syst Appl 37(12):8673-867

Li DF, Wu ZQ (2010) Generalized OWA operator based methodology for multiattribute decision-making using intuitionistic fuzzy sets. Crit View 4:13-34

Li DF (2011) The GOWA operator based approach to multiattribute decision making using intuitionistic fuzzy sets. Math Comput Modell 53(5-6):1182-1196

Liang C, Zhao S, Zhang J (2017) Multi-criteria group decision making method based on generalized intuitionistic trapezoidal fuzzy prioritized aggregation operators. Int J Mach Learn Cybern 8(2):597-610

Liu P, Chen SM, Liu J (2017) Multiple attribute group decision making based on intuitionistic fuzzy interaction partitioned Bonferroni mean operators. Inf Sci 411:98-121

Liu P, Liu X (2017) Multiattribute group decision making methods based on linguistic intuitionistic fuzzy power Bonferroni mean operators. Complexity 3:10

Liu P, Chen SM (2018) Multiattribute group decision making based on intuitionistic 2-tuple linguistic information. Inf Sci 430:599-619

Liu P, Liu J, Chen SM (2018) Some intuitionistic fuzzy Dombi Bonferroni mean operators and their application to multiattribute group decision making. J Oper Res Soc 69(1):1-24

Lu X, Ye J (2019) Similarity measures of linguistic cubic hesitant variables for multiple attribute group decision-making. Information 10(5): 168

Mahmood T, Mehmood F, Khan Q (2016) Cubic hesistant fuzzy sets and their applications to multi criteria decision making. Int $\mathrm{J}$ Algebra Stat 5:19-51

Nayagam VLG, Muralikrishnan S, Sivaraman G (2011) Multi-criteria decision-making method based on interval-valued intuitionistic fuzzy sets. Expert Syst Appl 38(3):1464-1467

Park JH, Gwak MG, Kwun YC (2011) Uncertain linguistic harmonic mean operators and their applications to multiple attribute group decision making. Computing 93(1):47

Phong PH, Cuong BC (2015) Max-Min Composition of Linguistic Intuitionistic Fuzzy Relations and Application in Medical Diagnosis. VNU J Sci Comput Sci Commun Eng 30:4

Rani D, Garg H (2018) Complex intuitionistic fuzzy power aggregation operators and their applications in multicriteria decisionmaking. Expert Syst 35(6): 12325

Riaz M, Tehrim ST (2019) Cubic bipolar fuzzy ordered weighted geometric aggregation operators and their application using internal and external cubic bipolar fuzzy data. Comput Appl Math 38(2):87

Szmidt E, Kacprzyk J (2000) Distances between intuitionistic fuzzy sets. Fuzzy Sets Syst 114(3):505-518

Shuqi W, Dengfeng L, Zhiqian W (2009) Generalized ordered weighted averaging operators based methods for MADM in intuitionistic fuzzy set setting. $J$ Syst Eng Electron 20(6): 1247-1254

Shakeel M (2018) Cubic averaging aggregation operators with multiple attributes group decision aking problem. J Biostat Biometric Appl 3(1):102

Wei GW (2009) Uncertain linguistic hybrid geometric mean operator and its application to group decision making under uncertain linguistic environment. Int J Uncertain Fuzziness Knowl Based Syst 17(02):251-267

Wei G, Zhao X, Lin R, Wang H (2013) Uncertain linguistic Bonferroni mean operators and their application to multiple attribute decision making. Appl Math Model 37(7):5277-5285

$\mathrm{Xu} \mathrm{Z} \mathrm{(2004)} \mathrm{Uncertain} \mathrm{linguistic} \mathrm{aggregation} \mathrm{operators} \mathrm{based}$ approach to multiple attribute group decision making under uncertain linguistic environment. Inf Sci 168(1-4):171-184

$\mathrm{Xu} Z$ Z (2004) A method based on linguistic aggregation operators for group decision making with linguistic preference relations. Inf Sci 166(1-4):19-30

$\mathrm{Xu} \mathrm{Z}$ (2006) A note on linguistic hybrid arithmetic averaging operator in multiple attribute group decision making with linguistic information. Group Decis Negot 15(6):593-604

$\mathrm{Xu} \mathrm{Z} \mathrm{(2006)} \mathrm{Induced} \mathrm{uncertain} \mathrm{linguistic} \mathrm{OWA} \mathrm{operators} \mathrm{applied} \mathrm{to}$ group decision making. Inf Fusion 7(2):231-238

Yager RR, Kacprzyk J, Beliakov G (2011) Recent developments in the ordered weighted averaging operators: theory and practice, vol 265. Springer, Berlin

Yang YR, Yuan S (2014) Induced interval-valued intuitionistic fuzzy Einstein ordered weighted geometric operator and their application to multiple attribute decision making. J Intell Fuzzy Syst 26(6):2945-2954

Ye J (2018) Multiple attribute decision-making method based on linguistic cubic variables. J Intell Fuzzy Syst 34(4):2351-2361

Zadeh LA (1965) Fuzzy sets. Inf Control 8(3):338-353

Zhao H, Xu Z, Ni M, Liu S (2010) Generalized aggregation operators for intuitionistic fuzzy sets. Int J Intell Syst 25(1):1-30

Zhou L, Chen H, Liu J (2013) Generalized multiple averaging operators and their applications to group decision making. Group Decis Negot 22(2):331-358

Zhou L, Chen H (2014) Generalized ordered weighted proportional averaging operator and its application to group decision making. Informatica 25(2):327-360

Zhang H (2015) Uncertain linguistic power geometric operators and their use in multiattribute group decision making. Math Problems Eng 3:10

Publisher's Note Springer Nature remains neutral with regard to jurisdictional claims in published maps and institutional affiliations. 